\title{
Adaptive sampling methodology for structural identification using
} radial-basis functions

\author{
Marco Proverbio ${ }^{12}$ \\ Alberto $\operatorname{Costa}^{3} 4$ \\ Ian F.C. Smith ${ }^{5}$, F. ASCE
}

\section{ABSTRACT}

The aim of model-based structural identification is to identify suitable models as well as values for model parameters that determine structure behaviour through comparing measurements with predictions. Well known methodologies, such as traditional implementations of Bayesian model updating, have been shown to be inaccurate in cases characterised by systematic uncertainties and unknown spatial correlations. Error-domain model falsification (EDMF) is another approach to structural identification. This approach is easy to understand for practising engineers and can provide robust parameter identification without assumptions on spatial correlations. The performance of all approaches involving sampling is affected by the number of model evaluations that is generated based on prior knowledge of parameter-value distributions. This paper focuses on a new sampling technique, called radial-basis function sampling (RBFS), and its application to EDMF, to generate a set of candidate models that represent the behaviour of the structure with a certain confidence level. RBFS provides a good exploration of the parameter space even with a limited number of samples, which results in reduced computation times. A full-scale bridge in Singapore has been tested and a new index of sampling quality is proposed in order to compare this approach

\footnotetext{
${ }^{1}$ ETH Zurich, Future Cities Laboratory, Singapore-ETH Centre, 1 CREATE Way, CREATE Tower, 138602 Singapore

${ }^{2}$ EPFL, Applied Computing and Mechanics Laboratory, CH-1015 Lausanne, Switzerland; E-mail: marco.proverbio@epfl.ch.

${ }^{3}$ National University of Singapore, Industrial \& Systems Engineering, Singapore.

${ }^{4}$ ETH Zurich, Future Resilient Systems, Singapore-ETH Centre, 1 CREATE Way, CREATE Tower, 138602 Singapore; E-mail: costa@lix.polytechnique.fr

${ }^{5}$ EPFL, Applied Computing and Mechanics Laboratory, CH-1015 Lausanne, Switzerland; E-mail: ian.smith@epfl.ch.
} 
with other sampling techniques such as Latin hypercube sampling (LHS) and Markov chain Monte Carlo (MCMC). Finally, a cross-validation method is employed to verify the robustness of the approach and the sensitivity of sampling on prediction reliability.

Keywords: adaptive sampling, radial-basis function, optimisation, structural model updating, error-domain model falsification, surrogate models.

\section{INTRODUCTION}

Existing infrastructure elements, which are often designed and built for fixed lifetimes, need to be maintained, retrofitted, adapted and replaced to meet new needs. The optimal planning of maintenance requires an accurate knowledge of how existing structures behave. This helps avoid, for example, replacement when structures have sufficient reserve. Also, expensive interventions may be avoided through implementation of cheaper and more sustainable alternatives.

Structural identification methods are used to improve knowledge of structural behaviour. Measurement data interpretation has been extensively employed for structural health monitoring in the last decades, as reviewed in (Catbas et al. 2013). Many researchers have studied model-free, sometimes called data driven, methods using data interpretation strategies (Posenato et al. 2010). While these methodologies may be interesting for damage detection, infrastructure future-proofing requires behaviour models to compare alternative scenarios and support decision-making. When structures are modelled, for instance using finite elements (FE), and measurements are carried out, model identification techniques are used to improve the accuracy of model predictions. Although advanced simulations require long computation times and sensor equipment may be expensive, quantifiable benefits arise when structural replacement and unnecessary interventions are avoided (Smith 2016).

Despite the fact that measurements provide additional information for assessment of structures, this inverse problem involves many assumptions and sources of uncertainties. Raphael and Smith (1998) proposed a multi-model approach, based on model falsification to overcome challenges associated with inverse problems. In this method, model-updating results consist of a set of candidate models that explain the measurements taken from a structure. Robert-Nicoud et al. 
(2005) applied the same methodology and determined threshold boundaries by combining model and measurement errors.

Goulet et al. (2013a) proposed a probabilistic extension, called error-domain model falsification (EDMF), for robust structural identification when there are systematic modelling uncertainties and when correlations between measurement locations are unknown. This methodology helps identify candidate models among an initial model population, generated according to prior knowledge and engineering judgment, by using measurement values and probabilistically determined thresholds to falsify incorrect model instances. First, uncertainties are combined and threshold bounds are evaluated according to a reliability of identification. Then all the instances for which residual values between predictions and measurements exceed these bounds, at one or more sensor locations, are rejected. Pasquier et al. (2015a) compared traditional Bayesian model updating and EDMF in terms of prediction accuracy and demonstrated that EDMF is more robust for both diagnosis and prognosis. Moreover, EDMF has been employed by Goulet et al. (2013b) to evaluate the serviceability-limit-state reserve capacity of the Langesand Bridge and by Pasquier et al. (2014) to evaluate the fatigue reserve capacity of the Aarwangen Bridge.

Structural identification methods work most efficiently when there is a prior identification of the most sensitive parameters for model identification and prediction. Parameters are selected using sensitivity analyses according to their relative importance on model predictions. Model predictions are obtained directly using either a FE solver or a surrogate model (SM), which are able to capture the essential behaviour of the real structure while being more efficient in terms of computation speed. Several families of SMs have been employed in structural engineering, such as response surfaces based on polynomial functions (Ren and Chen 2010), Kriging estimates (Simpson et al. 2001), and radial-basis functions (RBF) (Buhmann 2000). Surrogate models based on RBF often perform well for engineering applications (Holmström et al. 2008). Other methods such as neural networks may also be adopted. Neural networks are usually employed for regression and classification tasks and they often require large training sets to be effective. When using a neural network, the objective function is hidden inside its layered structure and an analysis of the 
relationship between parameters and objective function values becomes challenging.

In order to select representative sets of parameter values to be assigned to the initial model class, sampling techniques are employed. These model samples, also referred to as model instances, are usually evaluated using FE solvers. Uniform sampling techniques such as grid-based sampling (GBS) and Latin hypercube sampling (LHS) have been employed with EDMF (Goulet et al. 2010; Pasquier and Smith 2016). A direct stochastic algorithm, called PGSL (Raphael and Smith 2003), has been applied to identify candidate models (Robert-Nicoud et al. 2005). In Bayesian model updating, a well-established method for model updating is Markov chain Monte Carlo (MCMC), which is a variant of Monte Carlo (MC) sampling. MCMC is used to sample from the target distribution, which is proportional to the posterior distribution of parameter values.

Optimisation algorithms can be employed to increase sampling performance through focusing the search around a particular area of the parameter domain. Adaptive sampling algorithms generate new samples by learning from the feedback of the previous samples. In this case, the feedback is often based on the optimisation of an objective function.

The choice of the objective function affects algorithm performance. Gradient-based techniques have been applied successfully in the absence of multiple optima and when objective functions are smooth and continuous in order to identify parameters of statistical models. However, in structural identification, the objective function might have many local optima. In this case, stochastic techniques such as genetic algorithms, physics-inspired algorithms and swarm algorithms have been employed (Zhang et al. 2010a; Zhang et al. 2010b; Marwala 2010). These methods do not require gradient information and are easily implemented. However, good solutions are often obtained only by tailoring the method through parameter tuning and the convergence of this methods is typically slow (Conn et al. 2009). Hybrid approaches which consist of both a stochastic and a gradient-based optimisation have also been proposed (Christodoulou et al. 2008; Christodoulou and Papadimitriou 2007). A comprehensive overview of these methods and their application in the field of structural optimisation can be found in (Hare et al. 2013). Compared with non-gradient optimisation methods, derivative-free optimisation techniques have shown to converge to globally 
optimal solutions when enough evaluations are performed (Torn and Zilinskas 1989; Gutmann 2001). Some optimisation methods may converge towards a unique "optimal" solution. In structural identification, many models can explain the measurement data because of the inverse nature of the problem. The presence of measurement and modelling uncertainties increases ambiguity. Therefore, in order to increase identification accuracy, a range of good quality solutions is often preferred to a single optimal solution. The advantage of derivative-free optimisation approaches compared with most stochastic search algorithms is the computational efficiency, which is crucial when evaluations have a non-negligible computational cost.

The need of efficient methodologies for sampling in EDMF has already been highlighted in previous studies (Goulet and Smith 2013a; Pasquier and Smith 2016). Traditional sampling methods, such as GBS and LHS, are not able to exploit the knowledge acquired from samples that have been already evaluated. Stochastic search methods provide efficient sampling only when model evaluations can be computed fast - which is often not the case for complex models. Adaptive-sampling techniques based on surrogate model optimization and compatible with EDMF can enhance sampling accuracy while reducing computation times. Much work in this direction is still missing. In addition, EDMF requires initial model sets that represent the entire population of plausible solutions (i.e combination of parameter values). Inaccurate, biased or sparse sampling may lead to identification shortcomings. Therefore, the sensitivity of sampling on prediction reliability needs to be investigated.

Full-scale case studies are essential for validating model-updating methods since it is only at this scale that uncertainties show realistic magnitudes. Brownjohn et al. (2001) highlighted that field conditions affect the accuracy of measurements. Therefore, data collected during lab experiments may not be representative of measurements collected under ambient conditions (Catbas et al. 2013). Lam et al. (2015) proposed an application of MCMC to update the model of a coupled-slab system using field test data. They pointed out that, in real situations, the effects of both modelling error and measurement noise are relatively large when compared to numerical examples or experimental case studies under laboratory conditions. Therefore, posterior uncertainties when field data 
are collected are higher than those obtained using laboratory experiments.

While many full-scale studies have been carried out, few researchers have systematically validated data-interpretation proposals using full-scale structures (Simoen et al. 2013). Strong methods for result validation are required to assess the performance of model updating techniques. This study implements an approach where cross-validation is carried out using models that have been identified as suitable to predict at locations other than those used in model updating.

This paper proposes a new adaptive sampling methodology, to increase the performance of structural identification. The impact of the employed sampling methodology on prediction reliability is investigated. The first section provides background on error-domain model falsification and sampling algorithms. The subsequent section introduces the new sampling methodology and its application to EDMF. Finally, a full-scale case study is used to compare systematically the performance of the proposed approach with those of traditional sampling algorithms and to validate results of structural identification.

\section{BACKGROUND}

\section{Error-domain model falsification}

Initially proposed in (Goulet and Smith 2013a), EDMF helps identify plausible physics-based models using information provided by measurement data. Plausible models are defined by $n_{\theta}$ parameter values and a model class. Each model class $G_{k}$ has a unique parametrization that includes characteristics such as material properties, geometry, boundary conditions and actions.

Let $n_{y}$ be the number of measurement locations. For each location $i \in\left\{1, \ldots, n_{y}\right\}, R_{i}$ denotes the real responses of a structure (unknown in practice) and $\hat{y}_{i}$ corresponds to the measured value at location $i$. Predictions $g_{k}\left(x_{i}, \boldsymbol{\Theta}_{k}\right)$ of the model class $G_{k}$, which is usually based on a finite element analysis, are evaluated at location $x_{i}$ through assigning $\Theta_{k}$, which corresponds to instances of the parameter vector $\boldsymbol{\theta}_{k}$, to the model class. Since model-prediction uncertainty $U_{i, g_{k}}$ and measurement uncertainty $U_{i, \hat{y}}$ are unavoidable, model predictions and measurements are linked to the true 
behaviour using Equation (1).

$$
g_{k}\left(x_{i}, \Theta_{k}\right)+U_{i, g_{k}}=R_{i}=\hat{y}_{i}+U_{i, \hat{y}} \quad \forall i \in\left\{1, \ldots, n_{y}\right\}
$$

Rearranging the terms:

$$
g_{k}\left(x_{i}, \boldsymbol{\Theta}_{k}\right)-\hat{y}_{i}=U_{i, c}
$$

where $\boldsymbol{U}_{c}$ is a vector representing the difference between uncertainties $\boldsymbol{U}_{\hat{y}}$ and $\boldsymbol{U}_{g_{k}}$. The left-hand side of Equation (2) is the difference between a model prediction and a measurement at location $x_{i}$, which is often called the residual $r_{i}=g\left(x_{i}, \boldsymbol{\Theta}\right)-\hat{y}_{i}$.

The probability density function (PDF) describing the error in measurements $f_{\boldsymbol{U}_{\hat{y}}}\left(\boldsymbol{u}_{\hat{y}}\right)$ is usually estimated by conducting multiple series of tests under site conditions. Manufacturer specifications are often very optimistic lower bounds. The PDF describing the error in the model class $f_{\boldsymbol{U}_{g_{k}}}\left(\boldsymbol{u}_{g_{k}}\right)$ is estimated using values taken from the literature, stochastic methods, engineering judgment and local knowledge. In practical situations, uncertainties associated with the model class are usually much larger than measurement uncertainties. Thus, their quantification directly affects the performance of the method.

In the traditional implementation of EDMF, the identification process starts with the definition of an initial set of $n_{\Omega}$ model instances $\Omega_{k}=\left\{\boldsymbol{\Theta}_{k, m}, m=1, \ldots, n_{\Omega}\right\}$, usually through employing uniform sampling techniques. Then the instances for which the residual values exceed defined threshold boundaries are falsified.

In EDMF, threshold bounds are defined through computing the shortest interval $\left\{u_{i, l o w}, u_{i, h i g h}\right\}$ that contains a probability equal to $\phi_{d}^{1 / n_{y}}$ for the combined PDFs $f_{\boldsymbol{U}_{c}}\left(\boldsymbol{u}_{c}\right)$ at each sensor location, as expressed in the following equation:

$$
\phi_{d}^{1 / n_{y}}=\int_{u_{i, l o w}}^{u_{i, h i g h}} f_{U_{c, i}}\left(u_{c, i}\right) \mathrm{d} u_{c, i} \quad \forall i \in\left\{1, \ldots, n_{y}\right\}
$$

In Equation (3) the confidence level $\phi_{d}$ is adjusted using the Sidák correction to take into account 
that measurements at several locations are considered simultaneously to falsify model instances. The hyper-rectangular acceptance region adjusted with the Sidák correction (with dimensions corresponding to the number of sensors) has been shown to be conservative regardless of the value of correlation between sensor locations (Goulet and Smith 2013a). In the field of structural engineering, a value of 0.95 for the confidence level $\phi_{d} \in[0,1]$ is commonly employed. Falsification is then performed according to the following equation:

$$
\Omega_{k}^{\prime \prime}=\left\{\boldsymbol{\theta}_{k} \in \Omega_{k} \mid \forall i=1, \ldots, n_{y} \quad u_{i, l o w} \leq g_{k}\left(x_{i}, \boldsymbol{\theta}_{k}\right)-\hat{y}_{i} \leq u_{i, h i g h}\right\}
$$

where the candidate model set $(\mathrm{CMS}), \Omega_{k}^{\prime \prime}$, is made up of all the initial model instances except those that have been falsified at one or more measurement locations. An instance $\Theta$ of a model class $G$ is thus a candidate model if, for each sensor location $i \in\left\{1, \ldots, n_{y}\right\}$, the residual value lies inside the interval defined by the threshold boundaries.

Based on Equation (4), model instances that are falsified are assigned a null probability.

$$
\operatorname{Pr}\left(\Theta_{k} \notin \Omega_{k}^{\prime \prime}\right)=0
$$

Since knowledge of uncertainty-distribution forms is typically poor, all the model instances that belong to the CMS are assigned a constant probability:

$$
\operatorname{Pr}\left(\boldsymbol{\Theta}_{k} \in \Omega_{k}^{\prime \prime}\right)=\frac{1}{\int_{\theta_{k} \in \Omega_{k}^{\prime \prime}} \mathrm{d} \theta_{k}}
$$

It is very rare that a more sophisticated probability distribution for the CMS can be justified in practical situations.

When all initial model instances generated are falsified, the entire model class is falsified $\left(\Omega_{k}^{\prime \prime}=\emptyset\right)$. This means that no model is compatible with observations given the current estimation of model and measurement uncertainties. Thus it is usually a sign of incorrect assumptions in the model-class definition and uncertainty assumptions. Complete falsification helps avoid wrong 
identification of parameter values and detect flaws in initial assumptions, highlighting one of the main advantages of EDMF compared with other methodologies. The choice of the parameters to be identified is carried out by: i) conducting a sensitivity analysis to determine the relative importance of each parameter in model predictions and ii) considering the final goal of the structural identification, such as loading capacity estimation. For example, when serviceability is the critical limit state, relevant parameters include elastic material properties such as the Young's modulus and in-situ boundary conditions. In situations where the ultimate reserve capacity is investigated, relevant parameters include structural-element geometry and material strength values. The latter can be updated through non-destructive tests or laboratory tests. The choice of the initial interval and the distribution of parameter values to be adopted, usually uniform if no specific information is available, is based on engineering judgment. The parameters that most influence predictions are included in the vector of primary parameters $\boldsymbol{\theta}_{k}$ and used to generate the initial model set $\Omega_{k}$.

When a candidate model set is identified $\left(\Omega_{k}^{\prime \prime} \neq \emptyset\right)$, prediction tasks can be performed employing the CMS to predict at unmeasured locations and to assess the reserve capacity of the structure.

Predictions $Q_{j}$ at $n_{q}$ locations are given by:

$$
Q_{j}=g_{k}\left(x_{i}, \Theta_{k}^{\prime \prime}\right)+U_{i, g_{k}}, \quad \forall i \in\left\{1, \ldots, n_{q}\right\}
$$

where $\boldsymbol{\Theta}_{k}^{\prime \prime}=\left\{\boldsymbol{\Theta}_{k} \mid \boldsymbol{\theta}_{k} \in \Omega_{k}^{\prime \prime}\right\}$ is a set of vectors of parameter values representing the CMS.

There is a trade-off between the simplicity of the FE model and the magnitude of model uncertainty $U_{g_{k}}$. For example, it is possible to employ a less precise model class $G_{k}^{*}$, which is characterised by higher model-uncertainty magnitudes $\boldsymbol{U}_{g_{k}}^{*}$, to obtain more rapidly a good outcome compared with a detailed model. The "price to pay" in such cases is the loss of precision in prediction due to the higher variance of $Q_{j}^{*}$, according to Equation (7).

The performance of identification in reducing the initial parameter uncertainties depends on factors such as the initial choice of parameters, the sampling technique and the sensor configuration. Besides the selection of parameters to be considered as primary parameters, the generation 
of the initial model set (IMS) affects structural identification. The IMS should cover the parameter domain that defines candidate models with sufficient density, in order to provide unbiased predictions. Many sampling techniques have been adopted in structural model updating. Each of them involves a trade-off between density and extension of space exploration.

\section{Sampling techniques}

The goal of EDMF is to falsify incorrect model instances, whose population should represent adequately, for each model class, the uncertainty connected to the parameter values after measurement.

In previous applications of EDMF mainly uniform sampling techniques, such as grid-based sampling (GBS) and Latin hypercube sampling (LHS) have been adopted to explore the model instance solution space (Goulet et al. 2010; Pasquier and Smith 2016). The vector of parameter range $I_{j}=\left[\theta_{j, \text { low }}, \theta_{j, h i g h}\right] \quad \forall j \in\left\{1, \ldots, n_{\theta}\right\}$ where $\theta_{\text {low }}, \theta_{\text {high }}$ represent respectively the lower and upper bounds for each parameter, is defined based on engineering judgment and the distributions of parameter values are usually uniform within the range. Conservative large bounds for parameter range are often used in order to ensure that the correct solution is within bounds.

\section{Grid-based sampling}

In GBS model instances are generated according to an $n_{\theta}$-dimensional grid. Each parameter range is discretised into $\xi\left(I_{j}\right)$ intervals which define the density of the sampling. The IMS consists of a matrix having $n_{k}$ rows and $n_{y}$ columns, where the total number of combinations to be evaluated $n_{k}$, is calculated with the following equation:

$$
n_{k}=\prod_{j=1}^{n_{\theta}} \xi\left(I_{j}\right)
$$

For example, in a model class defined by 5 primary parameters, each of which is discretized in 8 uniform intervals, $8^{5}=32,768$ samples are evaluated. 
Latin hypercube sampling

LHS generates model instances in a square $n_{\theta}$-dimensional grid across the parameter space, whereby each sample is the only one in each axis-aligned hyperplane containing it. This algorithm represents a development of the Monte Carlo sampling methods and it is particularly adopted to avoid clustering of samples. LHS requires that each parameter is divided into the same number of intervals and the definition of the number of samples to be evaluated. The maximum number of combinations for a LHS of $M$ intervals and $n_{\theta}$ parameters can be computed with the following equation:

$$
\left(\prod_{n_{\theta}=0}^{M-1}\left(M-n_{\theta}\right)\right)^{n_{\theta}-1}=(M !)^{n_{\theta}-1}
$$

The main drawbacks of LHS are that extreme points, such as corners of the design space, are not necessarily covered, and that the selection of few samples can result in a poor exploration of the domain.

Optimal space filling

Optimal space-filling sampling (SF) is a method whereby LHS is extended with post-processing. SF is initialised as LHS and then optimised several times through maximising the distance between samples. Samples are equally distributed throughout the design space with the objective of gaining the maximum insight into the parameter domain with the fewest number of samples. SF shares some of the same drawbacks as LHS, though to a lesser degree. Possible disadvantages are that extreme points may not be covered and that a limited number of samples can result in a poor exploration of the domain.

Figure 1 shows, for example, a two-dimensional problem that has two input parameters. Twenty design intervals are considered and samples are generated using GBS, LHS and SF. Although GBS provides an extensive exploration of the domain, it requires the highest number of samples (i.e. 400). Through avoiding samples with common rows and columns, LHS and SF require only 20 samples. However, LHS may generate clustered samples and skip parts of the parameter domain. SF addresses extremes more effectively and provides a better coverage of the parameter domain. 
GBS is not feasible when many parameters have to be considered simultaneously because its sampling complexity is exponential with respect to the number of parameters. LHS and SFS involve strategies to reduce the number of samples compared with GBS while providing a good exploration of the parameter domain. However as with GBS, poor sample density is likely when high-dimension domains are investigated.

\section{Markov chain Monte Carlo}

Adaptive sampling techniques can be applied to increase the performance of the sampling especially when high-dimensional spaces are investigated. With non-adaptive sampling techniques, the IMS is first built based only on the parameter uncertainties. Adaptive sampling techniques require an iterative process because the choice of the next sample depends on the parameter domain already explored.

Markov chain Monte Carlo (MCMC) is an algorithm that samples from a target distribution that is proportional to the posterior PDF by constructing a random walk that has the desired distribution at equilibrium. The most common method to construct the random walk between subsequent states is the Metropolis-Hastings algorithm, which is extensively used in Bayesian model updating. Let $p(\theta)$ denote the target PDF and $q(\theta)$ the proposal density, which depends on the current state $k \in\left\{1, \ldots, n_{k}\right\}$. The algorithm proceeds as follows:

1. Sample from the proposal density: $q\left(\theta^{c} \mid \theta^{k}\right)$ to generate a candidate state $\theta^{c}$ from the previous state $\theta^{k}$;

2. Evaluate the ratio $p_{\text {accept }}=\frac{p\left(\theta^{c}\right) q\left(\theta^{k} \mid \theta^{c}\right)}{p\left(\theta^{k}\right) q\left(\theta^{c} \mid \theta^{k}\right)}$;

3. If $p_{\text {accept }} \geq 1, \theta^{c}$ is accepted. Otherwise, the proposal state is accepted with probability $p_{\text {accept }}<1$. If $\theta^{c}$ is accepted $\theta^{k+1} \leftarrow \theta^{c}$

4. A random number $r \in[0,1]$ is generated. If $p_{\text {accept }}>r$ the proposal state is accepted and $\theta^{k+1} \leftarrow \theta^{c}$, otherwise $\theta^{k+1} \leftarrow \theta^{k}$.

By generating random numbers and then comparing them with $p_{\text {accept }}$, it is possible to visit regions having high posterior probability relatively more often than those associated with low posterior 
probability. Additionally, even though the posterior had a maximum value and that point was reached during the exploration, the algorithm would keep building the posterior distribution until $n_{k}$ samples are generated.

The efficiency of this approach is affected by the choice of the proposal density function form and its optimal form is usually unknown beforehand. This issue is aggravated when the uncertainties are correlated and when the posterior PDF is peaked. Moreover, traditional MCMC techniques are inefficient to sample high-dimensional target PDF and cannot be applied if the PDF is multimodal (Ching and Chen 2007). These issues have already been studied for Bayesian model updating in (Beck and Au 2002), which proposed an adaptive Metropolis-Hastings (AMH) based on intermediate simpler PDFs instead of the target one, and later developed in (Ching and Chen 2007) which proposed a transitional version of MCMC (TMCMC). The latter method is based on the previous AMH but employs a re-sampling strategy, which is more robust against the increasing number of parameters, to generate the intermediate PDFs and it has been shown to perform well even when a peaked or multimodal posterior PDF has to be sampled. However, the main drawback of this approach resides in the number of intermediate stages required to go through all the adjacent PDFs, because the transition between one intermediate PDF and the next should be smooth, but more stages mean more samples to be evaluated.

Goulet (2012) applied a combination of MCMC and GBS to obtain a CMS compatible with EDMF using a likelihood function based on the k-order generalised Gaussian distribution. The number of samples required by MCMC to get the same CMS as GBS was found to be approximately $20 \%$ lower. A performance was evaluated based on the size of the CMS compared to the initial set and was evaluated only for a simple theoretical example, involving two parameters. Real case studies are characterised by many primary parameters (usually 5 to 10) and large initial parameter uncertainties, which provide conservative wide initial intervals for parameter values. As a consequence, MCMC performance is affected by high-dimensional target PDFs that have to be sampled and iterative strategies should be adopted to tailor the proposal distribution to the problem at hand. 
According to Equation (6), all the model instances inside the CMS are assigned the same probability, which means that the target PDF will be uniform. If the CMS consists of only few model instances, it is likely to observe a peaked posterior distribution, which can also be multimodal depending on the combination of parameter values generated while sampling the parameter space. In this circumstance, it is difficult to implement MCMC with Metropolis-Hastings because if the proposal PDF is wide and it is likely that the peaked region will be reached only by chance. If the proposal PDF is too narrow, the travel of the Markov chain will be slow and the peak could never be reached in a reasonable number of samples. Though this issue can be faced by an adaptive evaluation of the proposal distribution, sampling from a multimodal distribution may result in a Markov chain which is trapped in one local peak. Moreover, to increase the performance of identification and to provide redundant information, a large number of sensors, sometimes even more than the over-instrumentation limit, are employed to obtain measurement values. Although the multivariate Gaussian likelihood can be implemented to accommodate any number of measurement points, its evaluation can be difficult when many sensors are considered.

In this paper a real case-study is employed to compare sampling techniques. An alternative approach not explicitly related to Bayesian model updating that is appropriate for sampling highdimensional spaces through balancing domain exploration and result quality, and effective for low number of samples, is proposed. The basics of this method are presented in the next section.

\section{Derivative-free optimization}

In many engineering applications, the goal is to optimise an objective function whose analytical expression is unknown, and the function values are only available through a solver. In this study, the objective function is expressed in terms of the residual values between FE predictions and measurements. For complex structures, the solver evaluation is a time-expensive simulation and therefore, estimating the partial derivatives of the response surface by finite-difference methods within gradient-based methods is usually not convenient. Traditional derivative-free heuristics such as simulated annealing, genetic algorithms, and particle swarm optimisation are also not appropriate, since they often require many evaluations to find good quality solutions. Moreover, 
they may not converge to the best solution if not enough samples are evaluated. These methods have been used in structural optimisation, as reported in (Hare et al. 2013).

In general, a derivative-free optimization problem can be cast in the following form:

$$
\max f(\boldsymbol{\theta})
$$

$$
\boldsymbol{\theta} \in\left[\boldsymbol{\theta}^{L}, \boldsymbol{\theta}^{H}\right],
$$

where $\boldsymbol{\theta} \in \mathbb{R}^{n_{\theta}}$ is the vector of the parameters, whose lower and upper bounds are defined by the vectors $\boldsymbol{\theta}^{L} \in \mathbb{R}^{n_{\theta}}$ and $\boldsymbol{\theta}^{H} \in \mathbb{R}^{n_{\theta}}$, respectively, and $f: \mathbb{R}^{n_{\theta}} \rightarrow \mathbb{R}$ is the objective function to optimise. The key feature of Equation (10) is that the analytic expression of $f$ is unknown and the evaluation of the function value $f(\overline{\boldsymbol{\theta}})$, given a set of parameter values $\overline{\boldsymbol{\theta}}$, is provided only by a solver.

An alternative approach is to adopt a surrogate model of the function $f$. The kriging-based EGO (Efficient Global Optimization) method (Jones et al. 1998), the radial-basis function (RBF) method (Gutmann 2001), and the stochastic RBF method (Regis and Shoemaker 2007) implement this idea. These approaches build global models of the function $f$, and not local models that are employed by trust-region methods (Conn et al. 2009). This ensures a convergence to the global optimal solution, if the number of simulations is large enough, and allows identification of good quality solutions within a limited number of simulations. Previous studies (Holmström et al. 2008) have shown that the RBF method performs well on engineering problems.

\section{The radial-basis function method}

The goal of the radial-basis function method is to approximate the unknown objective function $f$ using predictions provided by a FE solver. The surrogate model of the objective function is an interpolant $s$ that is built by means of radial basis functions. The RBFs are special functions $\omega(\|\boldsymbol{\theta}-\overline{\boldsymbol{\theta}}\|): \mathbb{R}^{+} \rightarrow \mathbb{R}$ that depend on the Euclidean distance $r$ between a new set of parameter values $\boldsymbol{\theta}$ and parameter sets $\overline{\boldsymbol{\theta}}$ already employed in FE simulations.

There are several types of RBFs, and the most commonly employed in engineering are the linear $\operatorname{RBF} \omega(r)=r$, the cubic $\operatorname{RBF} \omega(r)=r^{3}$ and the thin plate spline $\operatorname{RBF} \omega(r)=r^{2} \log (r)$. 
Given $z$ parameter sets $\boldsymbol{\theta}_{1}, \ldots, \boldsymbol{\theta}_{z} \in\left[\boldsymbol{\theta}^{L}, \boldsymbol{\theta}^{H}\right]$ and the FEM predictions $f\left(\boldsymbol{\theta}_{i}\right)$, the RBF interpolant $s_{z}$ to the points $\left(\boldsymbol{\theta}_{i}, f\left(\boldsymbol{\theta}_{i}\right)\right), \forall i \in\{1, \ldots, z\}$ can be expressed as:

$$
s_{z}(\boldsymbol{\theta})=\sum_{i=1}^{z} \lambda_{i} \omega(r)+p(\boldsymbol{\theta}),
$$

where $\omega$ is the RBF employed, $p$ is a polynomial and $\lambda_{i} \in \mathbb{R}$ are the coefficients of the interpolant that are found by solving a linear system. The polynomial $p$ is needed to ensure the existence of the interpolant and depends on the RBF type. The polynomial guarantees that this system can be solved and that coefficients can be computed. Also, the minimum degree of the required polynomial depends on the RBF employed. For example, in the cubic and thin plate spline cases the polynomial must have a degree of at least 1 , whereas for the linear case a 0 -degree polynomial (i.e., a constant) is sufficient, as explained in (Costa and Nannicini 2015). Further details on the RBF method can be found in (Buhmann 2000).

According to the RBF method, after $z$ simulations a target value $f_{z}^{*}$ is set. The next set of parameter values $\boldsymbol{\theta}_{z+1}$ to be evaluated through the FE solver is the point in the domain which minimizes the bumpiness (see Figure 2) of the RBF interpolant if this new set $\left(\boldsymbol{\theta}_{z+1}, f_{z}^{*}\right)$ is considered. The RBF method requires the function $f$ to be smooth and an advantage of this methodology is that it is possible to obtain an analytical measure of the bumpiness (Costa and Nannicini 2015). Since smooth functions exhibit low bumpiness values, new parameter sets $\boldsymbol{\theta}_{z+1}$ are chosen through a bumpiness minimization procedure (further details are provided in the next section).

\section{RBF Optimization-RBFOpt}

RBFOpt is open-source software developed for radial-basis function optimization. The software and additional information are available in (Costa and Nannicini 2015). Using the notation introduced above, and by defining $z$ as the counter for the number of simulations and MAX_ITER as the maximum number of allowed simulations, the RBF method implemented by RBFOpt is summarized by the following steps:

1. Select and evaluate a set of $m$ starting points $S=\left\{\left(\boldsymbol{\theta}_{1}, f(\boldsymbol{\theta} 1)\right), \ldots,\left(\boldsymbol{\theta}_{m}, f\left(\boldsymbol{\theta}_{m}\right)\right)\right\}$; 
2. Set $z=m$ to include the simulations already performed;

3. Compute the RBF interpolant for the points in $S$ according to Equation (11);

4. Choose a target function value $f_{z}^{*}$;

5. Find the point $\boldsymbol{\theta}_{z+1}$ such that the interpolant to the points $S \cup\left(\boldsymbol{\theta}_{z+1}, f_{z}^{*}\right)$ is the least bumpy;

6. Evaluate $\boldsymbol{\theta}_{z+1}$ through the solver to obtain $f\left(\boldsymbol{\theta}_{z+1}\right)$;

7. Add $\left(\boldsymbol{\theta}_{z+1}, f\left(\boldsymbol{\theta}_{z+1}\right)\right)$ to $S$ and set $z=z+1$;

8. If $\mathrm{z}=\mathrm{MAX} \_$ITER stop; otherwise return to step 3.

The first step involves the initialization of the RBF interpolant, for which $m>n_{\theta}$ starting points are needed. Although many strategies may be employed to define the starting points, RBFOpt employs LHS to select $m=n_{\theta}+1$ random points.

The target value $f_{z}^{*}$ is chosen according to a cyclic strategy, which alternates between the exploration of unknown zones of the domain and the identification of good approximations obtained by the surrogate model. The target value is a guess of the best value that the real objective function may achieve. If this value is far from the current optima of the interpolant implies that the real objective function can achieve a much better value than those predicted by the interpolant. In this case, the points which can potentially yield this improvement are searched in unexplored part of the domain. When the target value is close to the optima of the current interpolant a good approximation of the real objective function is achieved, hence the next evaluation point will not be to far from points already sampled. Thus, cyclically changing the target value helps optimize the interpolant by avoiding local optimum traps. More details related to the target value definition can be found in (Costa and Nannicini 2015).

Figure 2 represents an illustrative example that describes the meaning of bumpiness. The blue circles are the points already evaluated $\boldsymbol{\theta}_{z}$. Considering a target value $f_{z}^{*}$, two predictions (interpolants red and green) can be obtained according to the choice of the next point $\boldsymbol{\theta}_{z+1}$. The green prediction is less bumpy and thus, it will be chosen by RBFOpt.

In summary, RBFOpt is an iterative algorithm that optimises an objective function, whose analytical expression is unknown. To build the surrogate model of the function $f$ (i.e. RBF in- 
terpolant) predictions provided by a FE solver are employed. Moreover, this method is able to provide good results rapidly, while other approaches such as neural networks, which often need a large training set to be effective, would be computationally expensive. Also, RBF returns an approximation of the objective function, thus allowing the option to explore other promising parts of the candidate domain. Potentials exist for RBFOpt to be applied to EDMF, using an appropriate objective function (the falsification function), for sampling purposes. The optimisation of this function helps increase sampling density in the candidate domain. This new approach is explained in the following sections.

\section{SURROGATE MODELS FOR ADAPTIVE SAMPLING}

FE models are often used to predict structural behaviour, given input data such as element geometry, material properties, boundary and interface conditions, load configurations, element type, mesh size, etc. To reduce modelling uncertainty, the mesh can be refined and shell or solid elements can be employed instead of linear elements. However, computation times increase. Surrogate models are substitutes for complex models, since they are able to capture the essential behaviour of a structure using much less computation time than FE models. A common approach is to build response surfaces based on polynomial functions, through minimising the least-square difference between response surface and FE model predictions. When surrogate models are adopted to simulate the structural behaviour, an additional source of uncertainty associated with the accuracy of the surrogate model has to be considered. Also, surrogate models may not represent adequately certain types of non-linear structural response.

Figure 3 shows a surrogate model that approximates the structural behaviour of a cantilever beam. The Young's modulus $E$ is the only unknown parameter. The goal is to predict the displacement $\delta$ at the free edge, under the distributed load $q$. The dashed hyperbolic line represents the true behaviour of the beam. Although in this simple case the mathematical formulation of $\delta^{T R U E}$ is well known, model predictions of real structures are usually provided by FE solvers. Since solver computation can be time consuming, two displacements $\left(\delta_{1}\right.$ and $\left.\delta_{2}\right)$ are evaluated using two values of parameter $E$. Then a linear surrogate model $\delta^{S M}$ is built and used to calculate the displacement 
$\delta$ for many samples of $E$.

The difference between the red line $\left(\delta^{S M}\right)$ and the dashed line $\left(\delta^{T R U E}\right)$ corresponds to the uncertainty of the surrogate model. Since the analytical formulation of the SM is built considering only few (in this example two) simulations, SM predictions may diverge from the true behaviour for E-values far from the two training points. This error can be reduced by increasing the density of training points and can be estimated by means of testing points, where both $\delta^{S M}$ and $\delta^{T R U E}$ are evaluated and compared. Increasing the number of training points enhances the accuracy of the SM; however, those points require time-consuming FE simulations.

Another implementation of surrogate models can be found in structural optimisation, as mentioned in the background section. The radial-basis function method - depicted in Figure 4 - is an adaptive sampling strategy that helps focus the sampling of parameter values in particular regions of the domain. Such a methodology can reduce the computation times required to reach a defined level of sampling accuracy. Considering the same cantilever beam as in the previous example, let assume that the goal is to identify E-values for which the residuals $\left(r=\delta-\delta^{*}\right)$ between predicted and measured maximum deflections lie in a predefined interval $\left[r_{l o w} ; r_{\text {high }}\right]$. This objective is described by means of a uniform residual target distribution $f(r)$. A Surrogate Model (SM) is used to generate samples of the parameter $E$, for which the residuals $r$ follow the residual target distribution. In Figure 4a $f(r)$ is defined in the prediction domain, while the analytic expression the function in the parameter domain $f(E)$ is unknown. Therefore, a SM $(R B F(E))$ is used to approximate the function $f$ in the parameter domain (Figure 4b). Then, the SM helps find target E-values - for which $f(E)=1$. Hence, the SM is used as an objective function to guide the sampling of $E$-values for which residuals follow the target distribution (Figure 4c). Compared with random sampling, the SM generates more samples (i.e. E-values) in the parameter sub-domain for which $f(E)=1$. Moreover, no SM uncertainty has to be considered, since every model prediction is computed using the FE solver. In this approach, the SM is used to approximate the unknown function $f(E)$. 


\section{RBF sampling for EDMF}

As mentioned above, surrogate models can be used for sampling purposes to help increase sampling density in sub-domains of the parameter space. The idea is to use radial-basis functions to create a surrogate model of a particular target distribution that characterises candidate models. This approach is applied to a new framework for adaptive sampling in EDMF, which is introduced in Figure 5.

This new methodology for sampling the parameter space is based on the optimisation of a surrogate model that represents a particular target distribution called the falsification function $\left(f_{F}\right)$. The optimization, performed using RBFOpt, helps provide sets of parameter values associated with residuals that lie within threshold bounds of falsification. The falsification function, which can be seen as the projection of a uniform likelihood function in the parameter space, is used as an objective function to guide the search of candidate models.

In order to compute the residuals $r_{i}$, model predictions are calculated using a FE solver and measurement values $y_{i}$ are collected from sensors. Furthermore, model and measurement uncertainties are combined to calculate the threshold bounds, as stated in Equation (2).

The next section provides a detailed explanation of the new framework.

\section{Iterative optimization}

In order to initialise the RBF, a set of parameter vectors $\tilde{\boldsymbol{\theta}}$ is defined by sampling in the initial parameter ranges $I_{j}$ using traditional techniques such as LHS. These vectors are assigned to the FE model class. The static analysis is performed using a FE solver such as ANSYS and predictions $g\left(x_{i}, \boldsymbol{\theta}\right)$ at locations $x_{i}$ are calculated. Then, residuals $r_{i}$ between measurement values and structural predictions are computed. According to Equation (2), the combined uncertainties $U_{i, c}$ and the residuals $r_{i}$ are compared in order to perform falsification. The falsification function, which is defined in the error domain, represents the target distribution. This distribution is approximated in the parameter domain by means of a surrogate model (i.e. RBF interpolant). The RBF interpolant is employed as an objective function for adaptive sampling. This improves the search for parameter values that provide residuals which follow the target distribution. 
RBFOpt generates the first RBF interpolant $r b f(\boldsymbol{\theta})$ which is a function $r b f: \mathbb{R}^{n_{\theta}} \rightarrow \mathbb{R}$ that provides the falsification value associated with the parameter values $\boldsymbol{\theta}$. The output of $r b f$-function are expressed in Equation (12)

$$
\operatorname{rbf}(\boldsymbol{\Theta})= \begin{cases}1 & , \text { if } \boldsymbol{\theta} \in \Omega^{\prime \prime} \\ {[0,1[} & , \text { otherwise }\end{cases}
$$

An instance $\Theta$ of the parameter vector $\theta$ that is in the candidate-model domain $\Omega^{\prime \prime}$, provides an interpolant value $\operatorname{rbf}(\boldsymbol{\Theta})=1$.

The generation of the interpolant consists of two steps. First, the interpolant type (i.e. linear, cubic) is chosen by a leave-one-out cross-validation and the RBF type that provides the smallest error is selected. Second, the choice of the interpolant shape is performed according to a bumpiness minimization procedure.

The RBF interpolant is iteratively optimised through selecting new instances $\Theta_{z+1}$, evaluating their predictions and computing the residuals. The choice of the next parameter sample is based on two criteria, the improvement of the interpolant accuracy and the search of the interpolant global maximum.

Satisfying these two criteria forms the core of the RBF sampling algorithm (RBFS). The improvement of the interpolant accuracy ensures a sufficient exploration of the parameter space while the search for the global maximum generates samples in the candidate domain. The framework in Figure 5 is performed iteratively until a stop or restart condition is reached. When the stop condition is reached, all model instances characterised by an RBF-value equal to 1 are automatically included in the CMS. Other instances are falsified.

The stop condition can be static or dynamic. In the former case, a maximum number of iterations is defined a priori, for instance, according to the available computation times. In the latter case, the variance of parameter values in the candidate domain is checked for each new candidate model. New model instances are generated until the variation in the variance is lower than a 
predefined limit.

A restart occurs when, after a given number of attempts, the current best solution (i.e. the maximum objective-function value $\operatorname{rbf}(\Theta)$ found so far) is not further improved. Consequently, a new random set of initial points is evaluated and a new RBF interpolant has to be optimised. This feature increases the exploration of the parameter domain. The entire process is summarised in the flowchart depicted in Figure 6.

\section{Falsification Function}

The falsification function is defined using the threshold boundaries computed through Equation (3). First, all the sources of uncertainties are combined using the Monte Carlo method and threshold bounds corresponding to $95 \%$ and $99 \%$ confidence level are calculated and corrected using the Sidák correction for a given number of measurements. The green area represents a rectangular distribution defined by the thresholds bounds $\left(T_{95, \text { low }} ; T_{95, \text { high }}\right)$. More formally, the function is built as follows:

$$
f_{F}\left(U_{i, c}\right)= \begin{cases}1 & , \text { if } u_{i, L}^{95}<U_{i, c}<u_{i, H}^{95}, \quad \forall i \\ 0 \quad, \text { otherwise } & \end{cases}
$$

where $u_{i, L}^{95}$ and $u_{i, H}^{95}$ represent the lower $(L)$ and upper $(H)$ threshold boundaries at sensor location $i$, calculated with a $95 \%$ confidence level. This confidence level is a standard engineering criterion that is used in many engineering decision tasks. Due to the systematic uncertainties in model-class definition, the $f_{F}$ is not centered in zero.

Since values of the falsification function for previously generated model instances are employed to fit RBF interpolants, a perfectly rectangular objective function is not appropriate because it cannot guide the search for optimal values. To guide the search, two triangular distributions are attached to the rectangular distribution, as shown in Figure 7. These distributions have a 0 probability at the $99 \%$ confidence bounds. The triangular distributions do not affect the falsification of model instances because all the samples outside the $95 \%$ interval (i.e. $f_{F}<1$ ) are discarded.

\section{CASE STUDY}


The case study is a prestressed reinforced concrete bridge in Singapore. This structure, which consists of four prestressed concrete beams, has a single span of $32 \mathrm{~m}$ and is supported at each end by 4 bearing devices (Figure 8 ). The beams support and are connected to a reinforced concrete slab that is $22 \mathrm{~cm}$ thick. The structure has been modelled using ANSYS and the model includes non-structural elements such as the asphalt pavement and precast concrete barriers. These elements, which are usually included as permanent loads in design-stage models, are included in order to reduce model-simplification uncertainties. A static load test involving 6 trucks, each with a gross weight of 33 tons, was performed. A measurement system consisting of a laser tracker, 8 strain gauges (S) and 2 inclinometers (I) has been designed. In order to increase the accuracy of deflection measurements, 4 prisms (P) were placed on the bottom faces of the main beams. Truck configuration and sensor locations are depicted in Figure 9.

\section{Uncertainty definition}

The case study is used to estimate the performance of RBFS and to compare it with results from LHS, SF and MCMC sampling. The parameters are defined according to a sensitivity analysis of parameter impact on model predictions at measurement locations. Figure 10 shows the relative importance of eight parameters that need to be defined in the FE model. Parameter selection is often a tradeoff - in this study the five most sensitive parameters are considered for identification. Although other parameters may provide additional insights, increasing the number of parameters for identification would result in a sampling domain with higher dimensions that requires larger sample sizes, in particular when non-adaptive sampling approaches are employed. In this study, the initial model set (IMS) is generated through sampling the five-dimensional parameter space defined by the Young's modulus of cast-in-place concrete, the Young's modulus of precast concrete, the Young's modulus of barrier concrete, the rotational and the vertical stiffness of the bearing devices.

The initial interval for each parameter, defined using the prior knowledge available, is reported in Table 1. This knowledge is based on a preliminary study and in-situ visual inspection. Intervals of elastic material properties (i.e. Young's moduli) include mean values from structural codes. Moreover, these intervals are conservatively wide in order to ensure that true parameter values 
are included. Rotational stiffness intervals are able to describe boundary conditions in the range between pinned and fixed constraint conditions. Vertical stiffness intervals are defined in order to consider vertical displacements of beam supports up to $40 \mathrm{~mm}$. Visual inspection did not provide evidence of local change of material properties. Therefore, constant properties have been assigned to every bridge element.

Table 2 describes modelling and measurement uncertainty sources. Uniform distributions are adopted to describe model uncertainty sources - a range of plausible assumptions on uncertainty forms are described in (Pasquier and Smith 2015b). The minimal and maximal bounds defined in Table 2 are expressed as a percentage of the mean value of model predictions for modelling uncertainties and as a percentage of the measured value or in absolute terms for measurement uncertainties. The uncertainty associated with the FE model takes into account two aspects: i) parameters that have not been considered for identification and ii) FE model simplifications. The variation in predictions due to parameters not considered for identification are estimated and included as uncertainties in model predictions. Model-simplification uncertainty is related to the FE-model-class features. In this study, the bridge model is constructed in ANSYS using solid elements (SOLID 185) with perfectly connected interfaces between adjacent layers. This assumption leads to a model class that is likely to overestimate the real stiffness of the bridge. Therefore, the FE uncertainty distribution is not centered on zero. Bounds of uncertainty associated with mesh refinement and bounds for additional uncertainty are taken from (Goulet et al. 2010). Finally, the source of uncertainty associated with spatial variability originates from strain sensors measuring behaviour that is affected by the spatial variability of material properties. This source compensates for the choice of assigning constant material properties to every bridge element. Uncertainty sources estimated in this paper are similar to those employed in previous studies that focus on bridges (Goulet et al. 2010; Pasquier and Smith 2016).

Sensor accuracies are described by uniform distributions that are based on manufacturer specifications. The measurement repeatability was assessed by taking multiple measurements under the same load case. Since the noise was mainly due to the vehicle traffic across a lane that was kept 
open during the test, a uniform distribution was used with bounds corresponding to the maximal and minimal values recorded. For strain gauges, uncertainty also arises from the imperfect alignment of gauges with respect to the bridge longitudinal axis, which results in underestimation of real stresses. Finally, additional noise associated with sensor installation has been considered for inclinometers and strain gauges using field observations and conservative engineering judgment. In order to perform model falsification, for each measurement location, a combined uncertainty PDF is computed and threshold bounds are determined for a confidence level fixed at $95 \%$. The remainder of this paper investigates the impact of sampling methodologies employed to generate the IMS on candidate-model identification.

\section{Candidate domain comparison (LHS, SF, MCMC and RBFS)}

Two non-adaptive sampling techniques (LHS and SF) and the traditional adaptive MCMC are compared with the new approach introduced in this paper (RBFS). Using each sampling technique, 1000 initial model instances are generated to form the initial model set. In order to assure a fair comparison, the same set of starting points is employed in RBFS and MCMC. Then, falsification is performed using threshold bounds and a candidate model set (CMS) is obtained using each sampling technique. Figure 11 shows the prediction values at each sensor location, which are referenced in accordance with Figure 9. Initial model instances are depicted in grey, while candidate models are highlighted by dark lines. The asterisks represent measured values taken by each sensor. LHS predictions are almost uniformly distributed throughout the parameter value range while RBFS predictions are denser near to measured values at each location. Moreover, RBFS provides more candidate models than LHS. RBFS provides larger prediction ranges than LHS at all sensor locations. This aspect helps avoid wrong identification due to the incomplete exploration of the prediction domain.

Figure 12 shows the parameter values that define model instances depicted in Figure 11. Both techniques cover the parameter domain. However, RBFS focuses the sampling of parameter values close to the candidate domain. For example, in Figure 12b sample density is denser for low values of the longitudinal stiffness and high values of precast concrete Young's modulus than in Figure 
12a. As shown in Figure 12c, RBFS increases the identified bounds for some parameters such as Young's modulus of cast-in-place concrete and the rotational stiffness. Moreover, for the rotational stiffness lower bound, RBFS helps identify a portion of the domain where LHS does not find candidate models. Similar observations can be made for the lower bound of cast-in-place concrete Young's modulus. The reduction of parameter initial range after falsification is related to characteristics of the measurement system. Some parameters such as the longitudinal stiffness and the precast Young's modulus are well identified, while for other parameters such as the Young's modulus of cast-in-place concrete, falsification does not reduce the initial interval. However, RBFS provides a thorough exploration of the candidate domain and helps avoid wrong falsification of parameter values that are caused by poor sampling.

\section{Sensitivity of initial model set size}

The number of samples that needs to be evaluated for reaching a defined level of performance is a crucial point for selecting the best sampling strategy. In the background section are mentioned some challenges, related to the sample size, in order to ensure a sufficient coverage of the parameter domain. Increasing the initial sample size is an effective way to improve the exploration. However, large sample sizes negatively affect evaluation times. For example, in this case study, the computation time required by ANSYS to solve one model instance is about 180 seconds using 12 cores in parallel (i.e. 50 hours for 1000 simulations). In order to compare RBFS with LHS, SF and MCMC, three initial sample sizes, which correspond to 500, 1000 and 2000 samples, are defined. Sample sizes smaller than 500 samples are not reasonable considering the dimension of the parameter domain and, thus, sampling performance would be largely affected by random generation of values. Sample sizes bigger than 2000 samples are discarded because of computation time constraints. Figures 13 and 14 present values of rotational stiffness and cast-in-place concrete Young's modulus that have been identified for three initial model sets that consist of 500, 1000 and 2000 samples.

Non-adaptive sampling techniques need many samples to provide a thorough exploration of the candidate domain while adaptive sampling techniques are more effective in identifying large 
parameter ranges using small numbers of model instances. For a large number of samples, the parameter ranges provided by the four sampling techniques are similar.

RBFS provides the highest number of candidate models regardless of the initial sample size. For the Young's modulus of cast-in-place concrete, RBFS is able to explore the entire candidate domain with 500 samples and outperforms MCMC for both sampling density and identification ranges. The employment of adaptive sampling techniques such as RBFS and MCMC increases the exploration of the candidate domain. In terms of parameter-value range for a fixed number of samples, RBFS often outperforms MCMC in exploring the candidate domain, especially when the number of samples is low.

In the next section, a quantitative index is defined to compare sampling algorithms.

\section{Quality index}

The quality index is a metric to compare the performance of sampling techniques in terms of parameter domain exploration. Let $k$ be a sampling technique. For each parameter $\theta$, the quality index at iteration $i$ (when $i$ model instances are generated) is defined as the ratio between i) the range of parameter values using sampling technique $k$ at iteration $i$ and ii) the range of parameter values obtained using all sampling techniques at the final iteration. Then, the values obtained for each parameter are averaged out. In this way, the quality index quantifies the increment of candidate domain exploration at each evaluation and for each sampling technique. This index is comparable to the performance profile used in derivative-free optimisation, where the performance of several algorithms is compared with the performance of the best algorithm (More and Wild 2009).

More formally, let $\mathrm{CMS}_{k}$ be the matrix of candidate models for the sampling technique $k$. $\mathrm{CMS}_{k}(t, \theta)$ represents the value of the parameter $\theta$ for the $t$-th model in the candidate model set. Let $\mathrm{CMS}_{\mathrm{T}}$ be the matrix obtained by merging the $\mathrm{CMS}_{k}$ for each $k$ and, if at iteration $i$ a candidate model is found, let $p(i)$ be its sequential position in the CMS. The quality index for the sampling technique $k$ at iteration $i$, indicated as $Q(k, i)$, is defined as follows: 


$$
Q(k, i)=\left\{\begin{array}{l}
0, \text { if } i=0, \\
Q(k, i-1), \text { if the } i \text {-th model does not belong to } \mathrm{CMS}_{k}, \\
\frac{1}{n_{\theta}} \sum_{\theta=1}^{n_{\theta}}\left(\frac{\max _{t \leq p(i)} \mathrm{CMS}_{k}(t, \theta)-\min _{t \leq p(i)} \mathrm{CMS}_{k}(t, \theta)}{\max _{t} \mathrm{CMS}_{\mathrm{T}}(t, \theta)-\min _{t} \mathrm{CMS}_{\mathrm{T}}(t, \theta)}\right), \text { otherwise. }
\end{array}\right.
$$

Figure 15 shows the comparison of the candidate domain generated using RBFS and four LHS. $\mathrm{LHS}_{0}$ denotes the default LHS-setting implemented in ANSYS. Three more populations Three more populations (LHS, $\mathrm{LHS}_{2}$ and $\mathrm{LHS}_{3}$ ) have been generated through a random selection of the sampling seed. The quality of candidate domain exploration provided by LHS is affected by the random choice of the seed value. However, there is no direct relationship between the choice of the seed value and the LHS performance. For example, although $\mathrm{LHS}_{3}$ is among the best of the LHS when the number of samples is less than 500, it provides the lowest quality of exploration when more than 1000 samples are generated. RBFS outperforms all sample seeds of LHS and most successful LHS seed at high-sample number $\left(\mathrm{LHS}_{1}\right)$ provides lower quality sampling compared with RBFS up to 1000 model samples. Moreover, RBFS is more likely to provide higher quality results than those provided by LHS, especially for low sample numbers $(<900)$.

As reported in the background section, MCMC requires the definition of a proposal distribution, which is usually Gaussian. A narrow proposal leads to inefficient sampling and many iterations to converge. A wide proposal may never find the candidate-model domain. A common approach is to keep adjusting the proposal width during iterations and to check the acceptance ratio of new samples. This strategy requires sample sizes large enough to ensure the convergence towards good width values. In this study, four proposal widths are employed to sample four IMSs, which consists of 2000 samples each. In this way, a fair comparison of sampling quality using 2000 samples can be carried out.

Figure 16 shows the quality index of RBFS and four MCMC characterised by different proposal widths. $\mathrm{MCMC}_{1}$ denotes the best parameter setting found after four attempts. The quality of candidate domain exploration provided by MCMC is affected by the proposal width employed. 
This is a drawback of this methodology since a good MCMC performance requires the tailoring of the proposal width to the problem at hand. However, the proposal width is not the only aspect to consider. The poor performance of the tested MCMC compared with RBFS is due to the limited number of samples. Indeed, after 2000 samples, MCMC has not converged. Additionally, the multi-dimensional posterior and the uniform likelihood adopted by EDMF reduce the ability of MCMC to focus the sampling in the candidate domain and to identify candidate models. In this case study, RBFS provides higher quality results than those provided by MCMC for all sample numbers. Moreover, since RBFS does not require initial tuning, there is less risk of low-quality results.

Figure 17 reports the final comparison between the four sampling techniques, in which the default value of the LHS seed is used $\left(\mathrm{LHS}_{0}\right)$. For MCMC, the best proposal width is chosen. Once again RBFS provides the best performance. After 2000 evaluations it performs the most accurate exploration of the candidate domain. Moreover, RBFS provides a quality of $75 \%$ in just 180 evaluations. For comparison, MCMC requires 630 iterations to reach the same quality level. For a limited number of FE evaluations (i.e. 500), RBFS provides the highest number of candidate models and the best exploration of the parameter domain. In this case, the quality index of RBFS is twice the one of LHS.

Moreover, RBFS requires the least number of samples to reach high levels of quality (one-fifth of the evaluations needed with MCMC for a quality index of 75\%). A 75\% quality is reached only after 1380 samples using SF and after more than 1800 samples using LHS.

\section{Cross-validation of structural identification}

Full-scale case studies are essential for validating model updating methods since it is only at this scale that uncertainties show realistic magnitudes. Unfortunately, many studies employ only simulated measurements. Likewise, data collected during lab experiments may not be representative of measurements collected under ambient conditions. Strong methods for result validation are required to assess the performance of model updating techniques. In this study, a cross-validation is carried out using candidate models that have been identified to predict at locations that were not 
used for model updating.

Figure 18 shows an example of cross-validation. First, 2000 initial samples are generated through RBFS. Then, falsification is performed using the threshold bounds. Predictions are obtained by combining model uncertainties with predictions of the candidate model set according to Equation (7). The CMS prediction distributions are shown (Figure 18). Because of the Sidák correction, the width of threshold bounds depends on the number of measurements that are used for falsification. Therefore, in order to perform the cross-validation, threshold bounds have to be calculated considering only the measurements that are used for falsification. Since use of EDMF involves the hypothesis that all candidate models are equivalently likely due to a lack of knowledge of real distribution forms, uniform prediction distributions (PDs) within 95\% threshold bounds are assigned to the CMS (Figure 18). The PDs at three locations (P1, S1 and I1), which have not been used for falsification, are plotted. For each sensor, the PD includes the measured value. Therefore, the cross-validation is verified and EDMF prognoses are robust.

Further investigation is carried out to assess the sensitivity of sampling on prediction reliability. Five sensors (three deflection prisms P2, P3, P4 and two inclinometers I1, I2) have not been used for falsification and one of the two inclinometers (I2) is used for cross-validation. Four sampling techniques (MCMC, LHS, RBFS, and SF) are employed to generate four IMSs of 500 and 1000 samples. The uniform PD at location I2 and the measured value are plotted in Figure 19. In this picture, the $y$-axis value of each uniform distribution is scaled to improve the visual interpretation of PDs. Table 3 summarizes cross-validation results.

The CMS provided by RBFS is validated when 500 initial samples are considered, while CMSs provided by MCMC and SF are validated only when the initial model set is large enough. Therefore, EDMF reliability can be affected by the exploration of the candidate domain provided by the adopted sampling algorithm. Interestingly, RBFS provides effective sampling even when the number of samples is low.

\section{SUMMARY AND CONCLUSIONS}

In this paper a new sampling methodology - referred to as RBFS - based on radial-basis func- 
tions (RBF) is employed in combination with error-domain model falsification (EDMF) to perform structural identification. First, the finite element model of a bridge is built, the most sensitive parameters are selected for sampling and uncertainties are quantified. Then, several sampling techniques are compared according to their performance in exploring the parameter domain while providing predictions that are compatible with the measured behaviour of the structure. RBFS overperforms traditional approaches (LHS and SF) and adaptive algorithms such as MCMC. Therefore, RBFS can increase the performance of EDMF by reducing computation times. Moreover, RBFS sampling accuracy helps avoid identification shortcoming and biased predictions.

Specific conclusions are as follows:

- Grid-based sampling is not feasible when many parameters are considered simultaneously. LHS and SFS involve a reduced number of samples; however, poor sample density is likely when high-dimensional domains are investigated.

- RBFS outperforms traditional uniform sampling techniques such as LHS and SF even for low numbers (500) of samples (FE evaluations). For the case study that was examined, RBFS provides the most effective exploration of the parameter domain and helps avoid the wrong falsification of parameter values connected with sampling shortcomings.

- The employment of adaptive sampling techniques such as RBFS and MCMC increases the exploration of the candidate domain. In terms of parameter-value range for a fixed number of samples, RBFS usually outperforms MCMC in exploring the candidate domain, especially when the number of samples is low.

- The quality index proposed in this paper is useful for comparing sampling techniques. When a quality level is established as a target (i.e. 75\%), RBFS requires the least number of samples. For example, MCMC may require more than four times the number of samples than RBFS to obtain the same quality level.

- EDMF prediction reliability is affected by the exploration of the candidate domain provided by the adopted sampling algorithm. RBFS provides effective sampling even when the number of initial samples is low. 
In order to generalise the conclusions above, other case studies involving real-scale measurements are ongoing. Future work will focus on assessing the performance of RBFS when more parameters are selected and when alternative definitions of model class and uncertainty magnitudes are considered. Also, further adaptive sampling algorithms have to be compared. Finally, the implication of sampling methodologies in the assessment of reserve capacity for existing bridges will be investigated.

\section{ACKNOWLEDGMENTS}

This research was conducted at the Future Cities Laboratory and the Future Resilient Systems at the Singapore-ETH Centre (SEC). The SEC was established as a collaboration between ETH Zurich and National Research Foundation (NRF) Singapore (FI 370074011-370074016) under the auspices of the NRF's Campus for Research Excellence and Technological Enterprise (CREATE) programme. The authors would like to gratefully acknowledge the support of the Land Transport Authority of Singapore (LTA) to perform the case study.

\section{REFERENCES}

Beck, J. L. and Au, S.-K. (2002). "Bayesian updating of structural models and reliability using markov chain monte carlo simulation.” Journal of Engineering Mechanics, 128(4), 380-391.

Brownjohn, J. M., Xia, P.-Q., Hao, H., and Xia, Y. (2001). "Civil structure condition assessment by fe model updating:: methodology and case studies." Finite elements in analysis and design, 37(10), 761-775.

Buhmann, M. D. (2000). “Radial basis functions.” Acta Numerica 2000, 9, 1-38.

Catbas, F. N., Kijewski-Correa, T. L., et al. (2013). "Structural identification of constructed systems." American Society of Civil Engineers,.

Ching, J. and Chen, Y.-C. (2007). "Transitional markov chain monte carlo method for bayesian model updating, model class selection, and model averaging." Journal of Engineering Mechanics, 133(7), 816-832.

Christodoulou, K., Ntotsios, E., Papadimitriou, C., and Panetsos, P. (2008). "Structural model 
updating and prediction variability using pareto optimal models." Computer Methods in Applied Mechanics and Engineering, 198(1), 138 - 149.

Christodoulou, K. and Papadimitriou, C. (2007). "Structural identification based on optimally weighted modal residuals.” Mechanical Systems and Signal Processing, 21(1), 4 - 23.

Conn, A., Scheinberg, K., and Vicente, L. (2009). Introduction to Derivative-Free Optimization. MOS-SIAM series on optimization. Society for Industrial and Applied Mathematics (SIAM, 3600 Market Street, Floor 6, Philadelphia, PA 19104).

Costa, A. and Nannicini, G. (2015). "RBFOpt: an open-source library for black-box optimization with costly function evaluations." Report No. 2014-09-4538, Optimization Online.

Goulet, J.-A. (2012). "Probabilistic model falsification for infrastructure diagnosis." PhD Thesis, n.5417, EPFL, Lausanne, Switzerland.

Goulet, J.-A., Kripakaran, P., and Smith, I. F. C. (2010). "Multimodel structural performance monitoring.” Journal of Structural Engineering, 136(10), 1309-1318.

Goulet, J.-A. and Smith, I. F. (2013a). "Structural identification with systematic errors and unknown uncertainty dependencies." Computers \& structures, 128, 251-258.

Goulet, J. A. and Smith, I. F. C. (2013b). "Performance-driven measurement system design for structural identification.” Journal of Computing in Civil Engineering, 27(4), 427-436.

Gutmann, H.-M. (2001). "A radial basis function method for global optimization.” Journal of Global Optimization, 19(3), 201-227.

Hare, W., Nutini, J., and Tesfamariam, S. (2013). "A survey of non-gradient optimization methods in structural engineering." Advances in Engineering Software, 59, 19 - 28.

Holmström, K., Quttineh, N.-H., and Edvall, M. M. (2008). "An adaptive radial basis algorithm (arbf) forexpensive black-box mixed-integer constrained global optimization." Optimization and Engineering, 9(4), 311-339.

Jones, D. R., Schonlau, M., and Welch, W. J. (1998). "Efficient global optimization of expensive black-box functions." Journal of Global Optimization, 13(4), 455-492.

Lam, H.-F., Yang, J., and Au, S.-K. (2015). "Bayesian model updating of a coupled-slab system 
using field test data utilizing an enhanced markov chain monte carlo simulation algorithm." Engineering Structures, 102, 144-155.

Marwala, T. (2010). "Finite-element-model updating using particle-swarm optimization.” Finiteelement-model Updating Using Computional Intelligence Techniques: Applications to Structural Dynamics, 67-84.

Moré, J. J. and Wild, S. M. (2009). “Benchmarking derivative-free optimization algorithms.” SIAM Journal on Optimization, 20(1), 172-191.

Pasquier, R., Goulet, J.-A., Acevedo, C., and Smith, I. F. C. (2014). "Improving fatigue evaluations of structures using in-service behavior measurement data." Journal of Bridge Engineering, 19(11), 04014045.

Pasquier, R. and Smith, I. F. (2015a). "Robust system identification and model predictions in the presence of systematic uncertainty." Advanced Engineering Informatics, 29(4), 1096 - 1109.

Pasquier, R. and Smith, I. F. (2015b). "Sources and forms of modelling uncertainties for structural identification." 7th International Conference on Structural Health Monitoring of Intelligent Infrastructure (SHMII), number EPFL-CONF-206997.

Pasquier, R. and Smith, I. F. (2016). "Iterative structural identification framework for evaluation of existing structures.” Engineering Structures, 106, 179 - 194.

Posenato, D., Kripakaran, P., Inaudi, D., and Smith, I. F. (2010). "Methodologies for model-free data interpretation of civil engineering structures." Computers \& structures, 88(7), 467-482.

Raphael, B. and Smith, I. (1998). "Finding the right model for bridge diagnosis." Artificial intelligence in structural engineering, Springer, 308-319.

Raphael, B. and Smith, I. (2003). "A direct stochastic algorithm for global search.” Applied Mathematics and computation, 146(2), 729-758.

Regis, R. G. and Shoemaker, C. A. (2007). "A stochastic radial basis function method for the global optimization of expensive functions.” INFORMS Journal on Computing, 19(4), 497-509.

Ren, W.-X. and Chen, H.-B. (2010). "Finite element model updating in structural dynamics by using the response surface method.” Engineering Structures, 32(8), 2455 - 2465. 
Robert-Nicoud, Y., Raphael, B., and Smith, I. F. (2005). "System identification through model composition and stochastic search." Journal of Computing in Civil Engineering, 19(3), 239247.

Simoen, E., Moaveni, B., Conte, J. P., and Lombaert, G. (2013). "Uncertainty quantification in the assessment of progressive damage in a 7-story full-scale building slice.” Journal of Engineering Mechanics, 139(12), 1818-1830.

Simpson, T., Mauery, T., Korte, J., and Mistree, F. (2001). "Kriging models for global approximation in simulation-based multidisciplinary design optimization.” AIAA Journal, 39(12), 22332241.

Smith, I. F. (2016). "Studies of sensor-data interpretation for asset management of the built environment." Frontiers in Built Environment, 2, 8.

Torn, A. and Zilinskas, A. (1989). Global Optimization. Springer-Verlag New York, Inc., New York, NY, USA.

Zhang, Z., Koh, C., and Duan, W. (2010a). "Uniformly sampled genetic algorithm with gradient search for structural identification-part i: Global search.” Computers \& structures, 88(15), 949962.

Zhang, Z., Koh, C., and Duan, W. (2010b). "Uniformly sampled genetic algorithm with gradient search for structural identification-part ii: Local search.” Computers \& structures, 88(19), 1149_ 1161. 


\section{List of Tables}

1 Parameter initial intervals. . . . . . . . . . . . . . . . 37

2 Modelling and measurement uncertainty sources. . . . . . . . . . . . . 38

3 Outcome of prediction cross-validation at location I2 ( $\checkmark$ : successful cross-validation, $\times$ : not successful cross-validation). . . . . . . . . . . . . . . . . . 39 


\begin{tabular}{lll}
\hline Parameters & Lower bound & Upper bound \\
\hline Young's modulus of cast-in-place concrete & $20 \mathrm{GPa}$ & $35 \mathrm{GPa}$ \\
Young's modulus of precast concrete & $25 \mathrm{GPa}$ & $50 \mathrm{GPa}$ \\
Young's modulus of barrier concrete & $3 \mathrm{GPa}$ & $40 \mathrm{GPa}$ \\
Rotational stiffness of bearing devices & $9 \log (\mathrm{Nmm} / \mathrm{rad})$ & $13 \log (\mathrm{Nmm} / \mathrm{rad})$ \\
Vertical stiffness of bearing devices & $8 \log (\mathrm{N} / \mathrm{mm})$ & $11 \log (\mathrm{N} / \mathrm{mm})$ \\
\hline
\end{tabular}

TABLE 1. Parameter initial intervals. 


\begin{tabular}{lllllll}
\hline Uncertainty source & \multicolumn{2}{c}{ Displacements - (P) } & \multicolumn{2}{c}{ Rotations - (I) } & \multicolumn{2}{c}{ Strains - (S) } \\
\hline & Min & Max & Min & Max & Min & Max \\
\hline FE model (\%) & -5 & 13 & -5 & 13 & -5 & 13 \\
Mesh refinement (\%) & -1 & 1 & -1 & 1 & -1 & 1 \\
Spatial variability (\%) & - & - & - & - & -5 & 5 \\
Additional uncertainty (\%) & -1 & 1 & -1 & 1 & -1 & 1 \\
\hline Sensor accuracy & $-0.05 \mathrm{~mm}$ & $0.05 \mathrm{~mm}$ & $-1 \mu \mathrm{rad}$ & $1 \mu \mathrm{rad}$ & $-2 \mu \epsilon$ & $2 \mu \epsilon$ \\
Repeatability & $-0.15 \mathrm{~mm}$ & $0.15 \mathrm{~mm}$ & $-4 \mu \mathrm{rad}$ & $4 \mu \mathrm{rad}$ & $-4 \mu \epsilon$ & $4 \mu \epsilon$ \\
Sensor orientation $(\%)$ & - & - & - & - & 0 & 6 \\
Sensor installation $(\%)$ & - & - & -5 & 5 & 0 & 5 \\
\hline
\end{tabular}

TABLE 2. Modelling and measurement uncertainty sources. 


\begin{tabular}{lcc}
\hline Sampling technique & Number of initial samples \\
\hline Optimal space filling - SF & 500 & 1000 \\
Latin hypercube sampling - LHS & $\times$ & $\checkmark$ \\
Markov chain Monte Carlo - MCMC & $\times$ & $\times$ \\
Radial-basis function sampling - RBFS & $\checkmark$ & $\checkmark$ \\
\hline
\end{tabular}

TABLE 3. Outcome of prediction cross-validation at location 12 ( $\checkmark$ : successful cross-validation, $\times$ : not successful cross-validation). 


\section{List of Figures}

1 Examples of sampling in a 2D domain using: a) grid-based sampling, b) Latin hypercube sampling (LHS) and c) optimal space-filling sampling (SF). . . . . . . . 43

2 Interpolant selection according to the bumpiness minimization criterion. The blue circles are the points already evaluated $\boldsymbol{\theta}_{z}$. Considering a target value $f_{z}^{*}$ (dashed line), two interpolants (red and green) can be obtained according to the choice of the next point $\boldsymbol{\theta}_{z+1}$ (square). The green interpolant is less bumpy. . . . . . . . . . . 44

3 Surrogate-model of the structural behaviour. A Surrogate models (SM) is used to speed up the computation of the maximum deflection $\delta$ in a cantilever beam. To build the SM, first two predictions are provided by the FE solver (blue points). Then, additional predictions are calculated using the SM since it computes faster than the FE solver. . . . . . . . . . . . . . . . . . 45

4 Surrogate-model of the target distribution. A Surrogate Model (SM) is used to generate samples of the parameter $E$, for which the residuals $\left(r=\delta-\delta^{*}\right)$ between predicted and measured maximum deflections follow the uniform target distribution. The residual target distribution $f(r)$ is defined in the prediction domain a), while the analytic expression the function in the parameter domain $f(E)$ is unknown. Therefore, a SM $(R B F(E))$ is used to approximate the function $f$ in the parameter domain b). Then, the SM helps find target E-values for which $f(E)=1$. Hence, the $\mathrm{SM}$ is used as an objective function to guide the sampling of $E$-values for which residuals follow the target distribution c). . . . . . . . . . . . 46

5 General framework of the radial-basis function sampling (RBFS). $\left(^{*}\right)$ for the Falsification Function refer to Figure $7 \ldots \ldots$. . . . . . . . . . . 47

6 Flow chart of the radial-basis function sampling (RBFS). $\left({ }^{*}\right)$ for the Falsification Function refer to Figure $7 \ldots \ldots \ldots \ldots$. . . . . . . . . . 48 
7 The falsification function is defined by the combined uncertainties. Two triangular distributions are attached to the rectangular distribution to guide the search for optimal values. . . . . . . . . . . . . . . . . . . . . 49

8 Plan view a), cross-section b) and longitudinal profile c) of the bridge. The flyover is a 32-meter-long span reinforced-concrete bridge that consists of four precast beams with cast-in-place diaphragms at the abutments. The cast-in-place concrete deck connects all the beams and the two precast concrete barriers. . . . . . . . . . 50

9 Top and bottom views of the flyover. The truck configuration is shown along with the position of two inclinometers (I), four deflection prisms (P) and eight strain

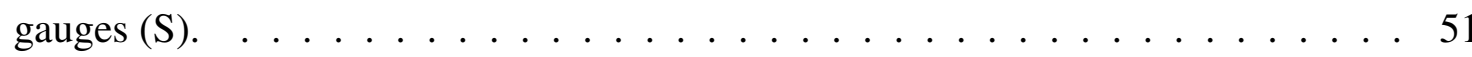

10 Relative importance of parameters $\boldsymbol{\theta}$ on model predictions at three sensor locations

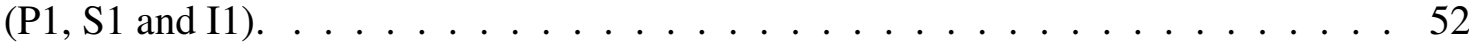

11 Predictions of 1000 model instances generated using Latin hypercube sampling (LHS), top and radial basis function sampling (RBFS), bottom. Each vertical axis represents the prediction at the sensor locations defined in Figure 9. Predictions of the initial model set (IMS) (grey lines) and the candidate model set (CMS) (dark lines) are plotted. CMS-prediction thresholds are reported for each axis. Black asterisks $(*)$ represent the measured value at each location. . . . . . . . . . . . 53

12 Parameter values describing 1000 model instances generated using LHS and RBFS. Each vertical axis represents a parameter. Initial model instances (grey lines) and candidate models (dark lines) are plotted and initial values of each parameter are given for each axis. The comparison of candidate models is shown in c). . . . . . . 54

13 Comparison of rotational stiffness values identified using RBFS, MCMC, LHS and SF. Falsification has been conducted considering three initial model sets that consist of 500, 1000 and 2000 samples. RBFS provides the largest CMS and the most effective exploration of the candidate domain, even with 500 samples. . . . . 55 
14 Comparison of Young's modulus values for cast-in-place concrete, identified using RBFS, MCMC, LHS and SF. Falsification has been conducted considering three initial model sets that consist of 500, 1000 and 2000 samples. RBFS covers the entire candidate domain within 500 samples, performing better than MCMC. . . . 56

15 Comparison of the candidate domain provided by 2000 samples generated using RBFS and four LHS. LHS 0 denotes the default LHS-setting implemented in ANSYS. Three more populations $\left(\mathrm{LHS}_{1}, \mathrm{LHS}_{2}\right.$ and $\mathrm{LHS}_{3}$ ) have been generated through a random selection of the sampling seed. The quality of candidate domain exploration provided by LHS is affected by the random choice of the seed value. . . 57

16 Comparison of the candidate domain provided by 2000 samples generated using RBFS and four MCMC characterised by different proposal widths. $\mathrm{MCMC}_{1}$ denotes the best parameter setting found after four attempts. Since RBFS does not require initial tuning, there is less risk of low-quality results. . . . . . . . . . . 58

17 Comparison of the candidate domain provided by 2000 samples generated using RBFS, $\mathrm{LHS}_{0}, \mathrm{MCMC}_{1}$ and SF. The main drawback of non-adaptive sampling is the slow exploration of the domain with increasing number of samples. . . . . . . 59

18 Distribution of candidate-model-set predictions (PD) at three sensor locations (P1, S1 and I1) that have not been used for falsification. The uniform PDs for a 95\% confidence and the CMS prediction distributions are plotted. For each sensor, the PD includes the measured value. The PDs have been identified using 2000 initial samples generated through RBFS. . . . . . . . . . . . . . . . 60

19 Uniform distributions of candidate-model-set predictions (PD), for a $95 \%$ confidence, at sensor location I2. Sensors P2, P3, P4, I1 and I2 have not been used for falsification and two PDs have been identified using respectively 500 (a) and 1000 (b) initial samples. The measurement value at location I2 is shown (dashed line). . 61 
a) Grid-based sampling (400 samples) b) Latin hypercube sampling (20 samples)

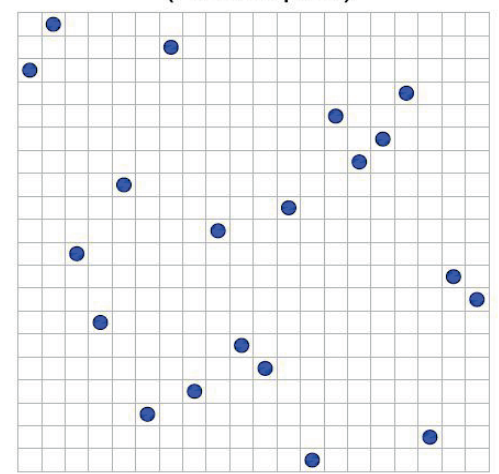

c) Optimal space filling (20 samples)

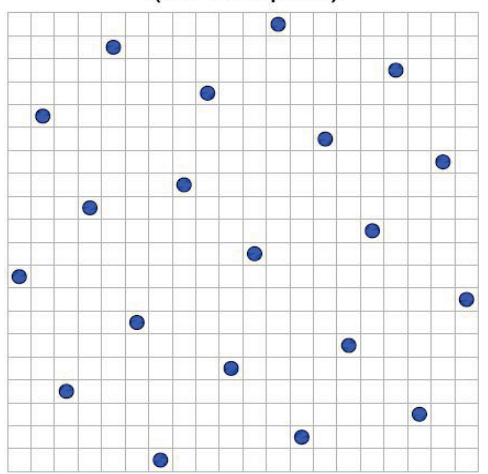

FIG. 1. Examples of sampling in a 2D domain using: a) grid-based sampling, b) Latin hypercube sampling (LHS) and c) optimal space-filling sampling (SF). 


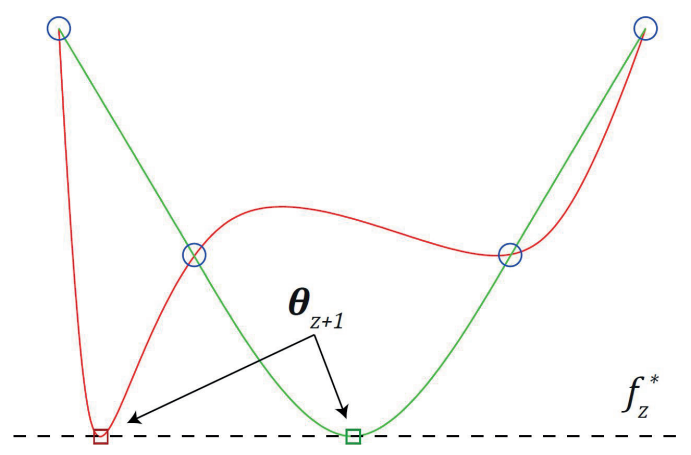

FIG. 2. Interpolant selection according to the bumpiness minimization criterion. The blue circles are the points already evaluated $\theta_{z}$. Considering a target value $f_{z}^{*}$ (dashed line), two interpolants (red and green) can be obtained according to the choice of the next point $\theta_{z+1}$ (square). The green interpolant is less bumpy. 


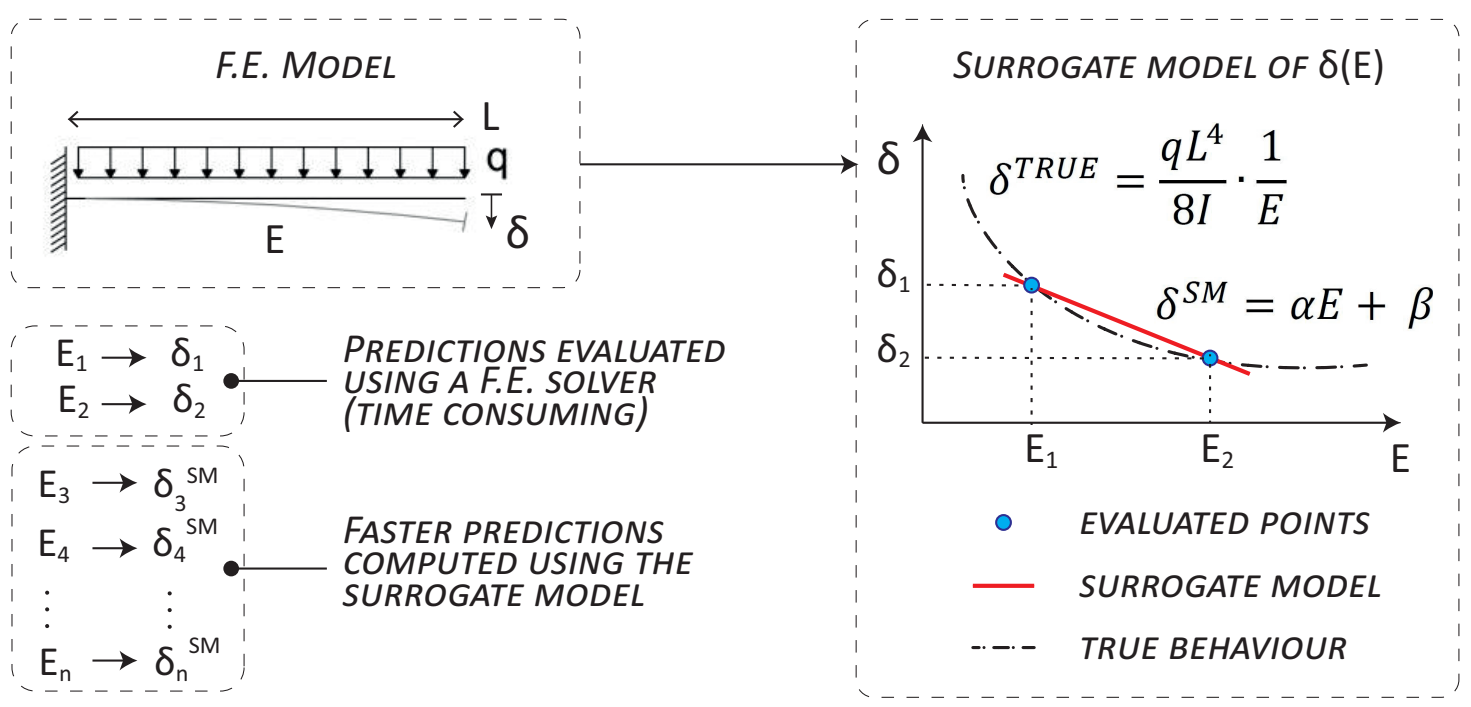

FIG. 3. Surrogate-model of the structural behaviour. A Surrogate models (SM) is used to speed up the computation of the maximum deflection $\delta$ in a cantilever beam. To build the SM, first two predictions are provided by the FE solver (blue points). Then, additional predictions are calculated using the SM since it computes faster than the FE solver. 


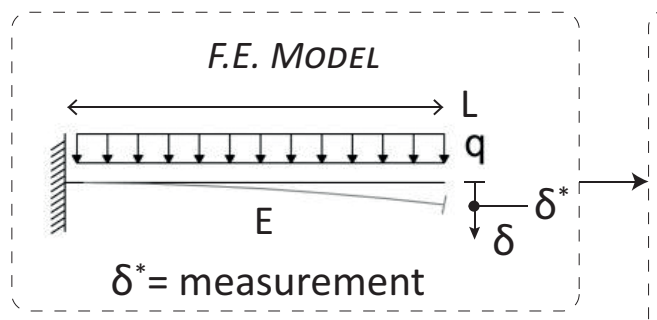

a) RESIDUAL TARGET DISTRIBUTION

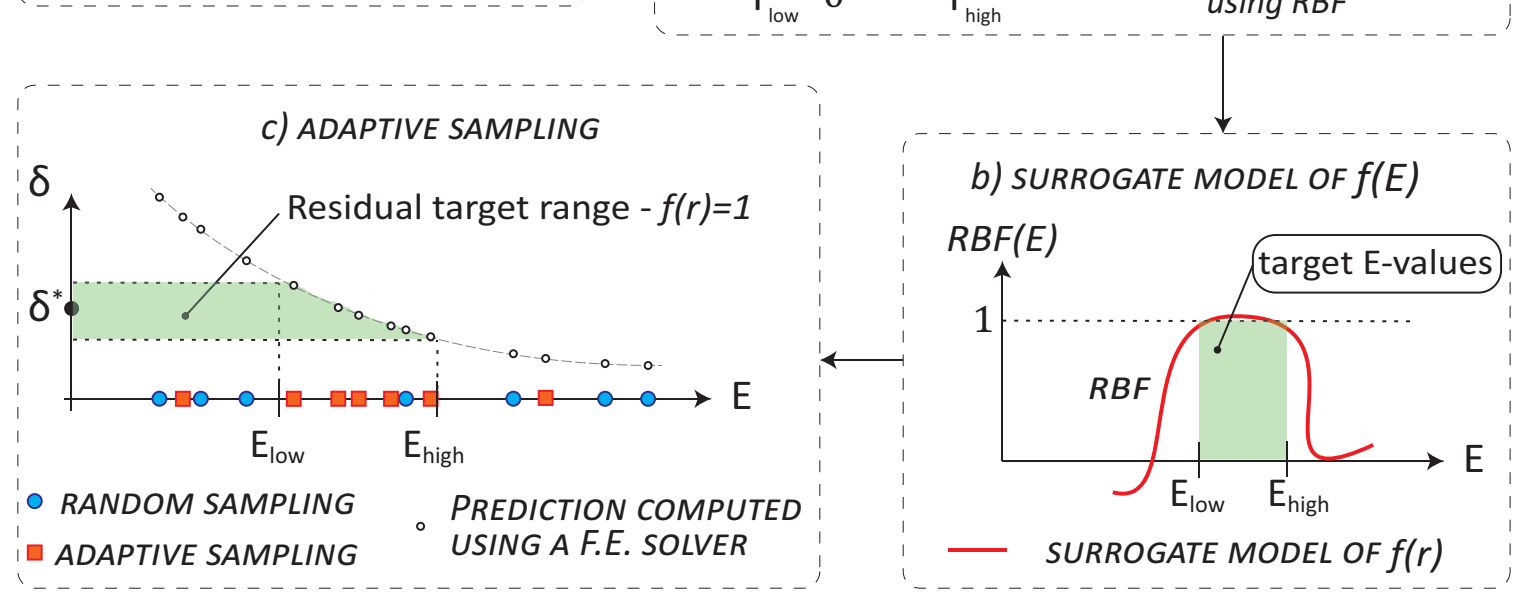

FIG. 4. Surrogate-model of the target distribution. A Surrogate Model (SM) is used to generate samples of the parameter $E$, for which the residuals $\left(r=\delta-\delta^{*}\right)$ between predicted and measured maximum deflections follow the uniform target distribution. The residual target distribution $f(r)$ is defined in the prediction domain a), while the analytic expression the function in the parameter domain $f(E)$ is unknown. Therefore, a SM $(R B F(E))$ is used to approximate the function $f$ in the parameter domain b). Then, the SM helps find target E-values for which $f(E)=1$. Hence, the SM is used as an objective function to guide the sampling of $E$-values for which residuals follow the target distribution c). 


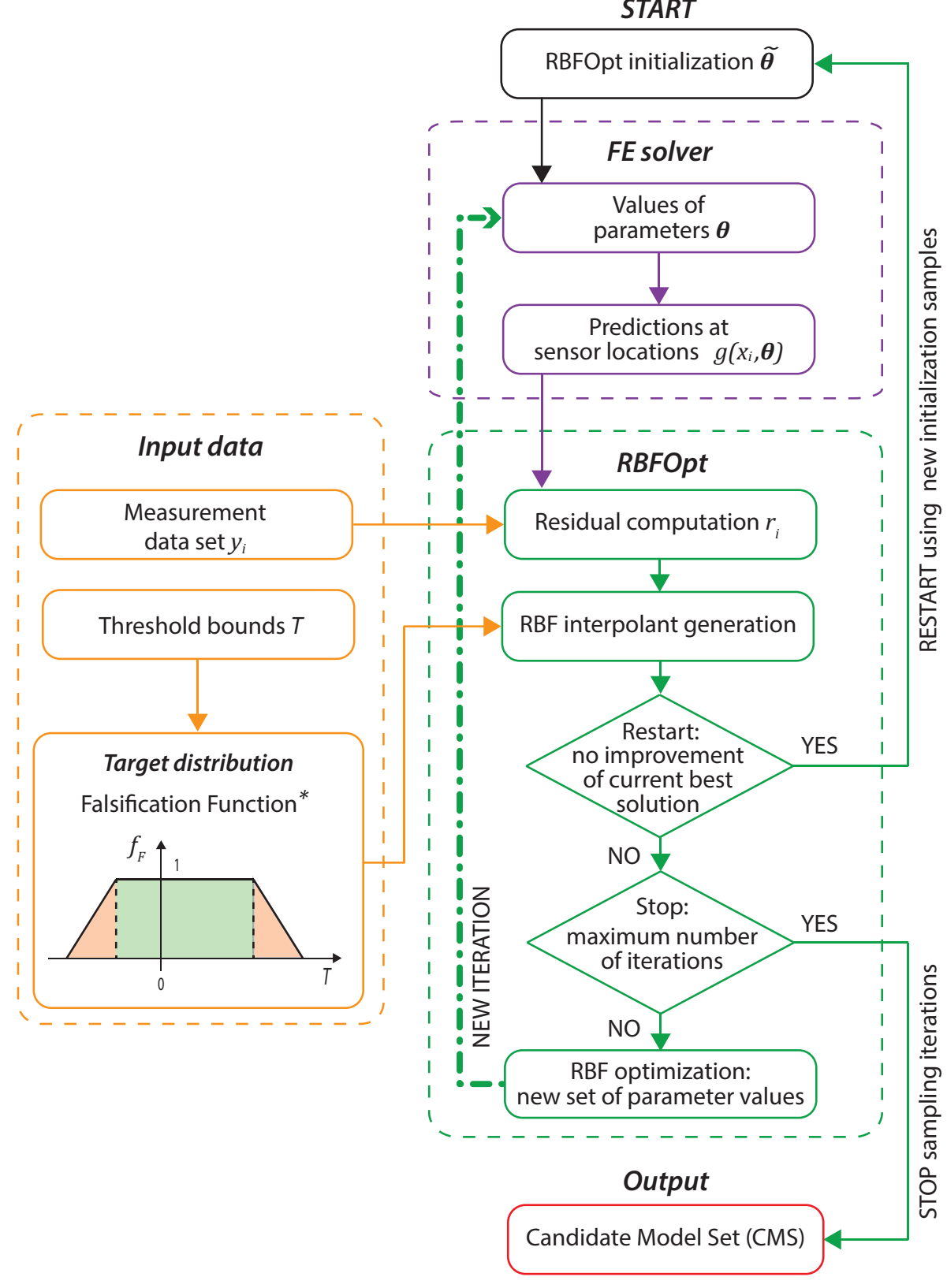

FIG. 5. General framework of the radial-basis function sampling (RBFS). (*) For the Falsification Function refer to Figure 7. 


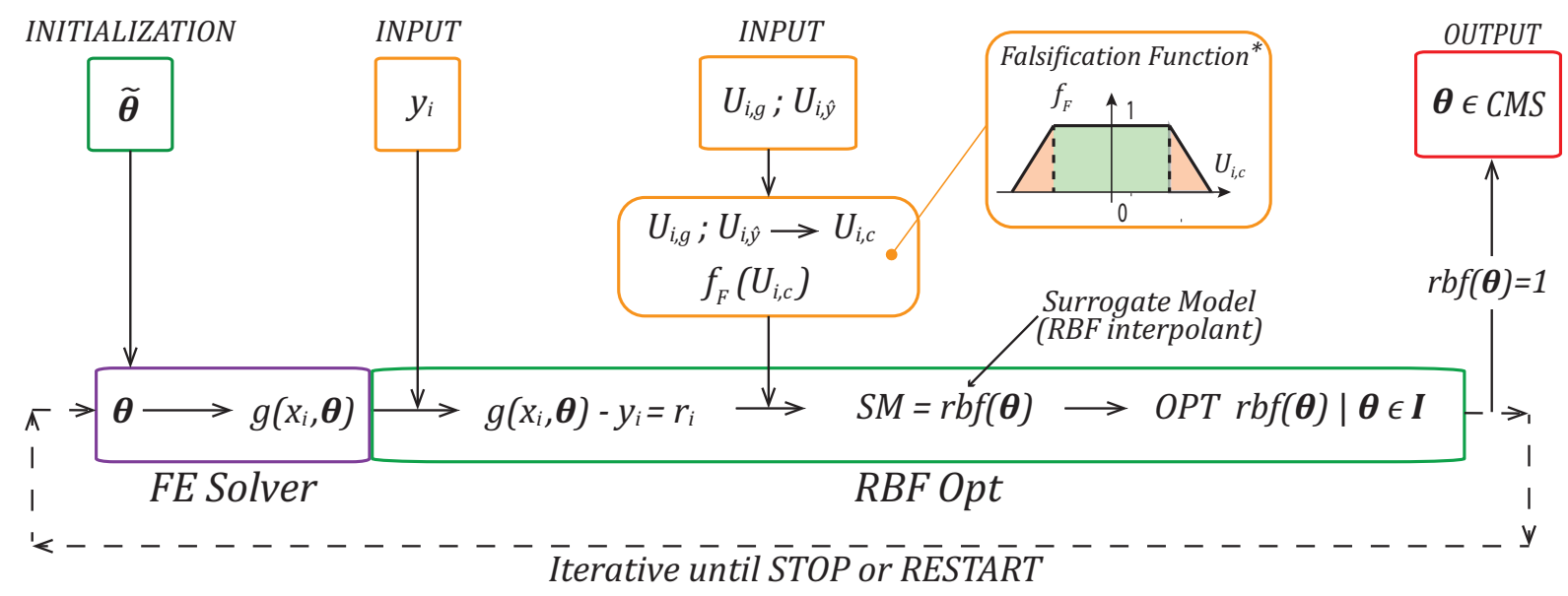

FIG. 6. Flow chart of the radial-basis function sampling (RBFS). $\left({ }^{*}\right)$ For the Falsification Function refer to Figure 7. 


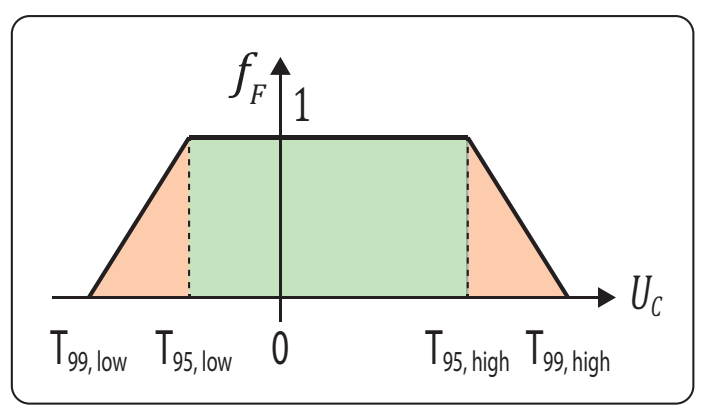

FIG. 7. The falsification function is defined by the combined uncertainties. Two triangular distributions are attached to the rectangular distribution to guide the search for optimal values. 


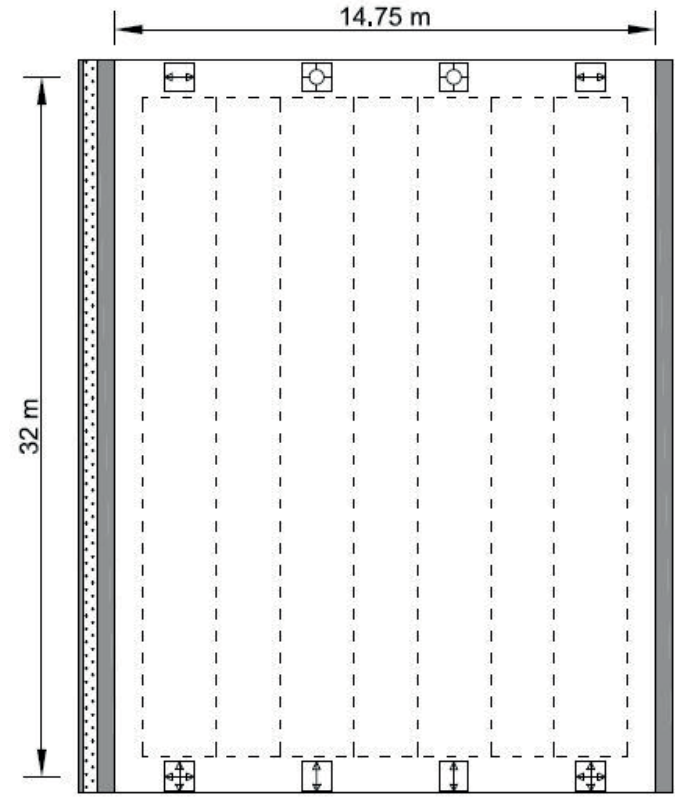

a) plan view
Bearing devices:

Free sllding

$\Theta$ Guided bearing

회 Fixed bearing

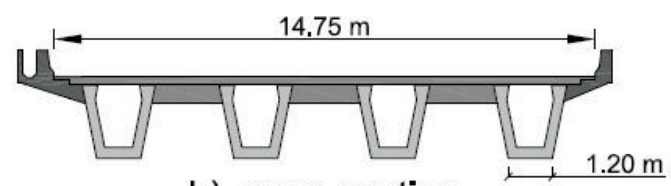

b) cross-section

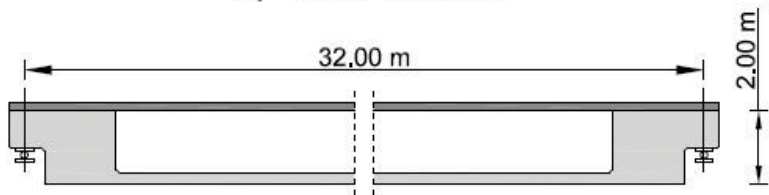

c) elevation

FIG. 8. Plan view a), cross-section b) and longitudinal profile c) of the bridge. The flyover is a 32-meter-long span reinforced-concrete bridge that consists of four precast beams with cast-in-place diaphragms at the abutments. The cast-in-place concrete deck connects all the beams and the two precast concrete barriers. 

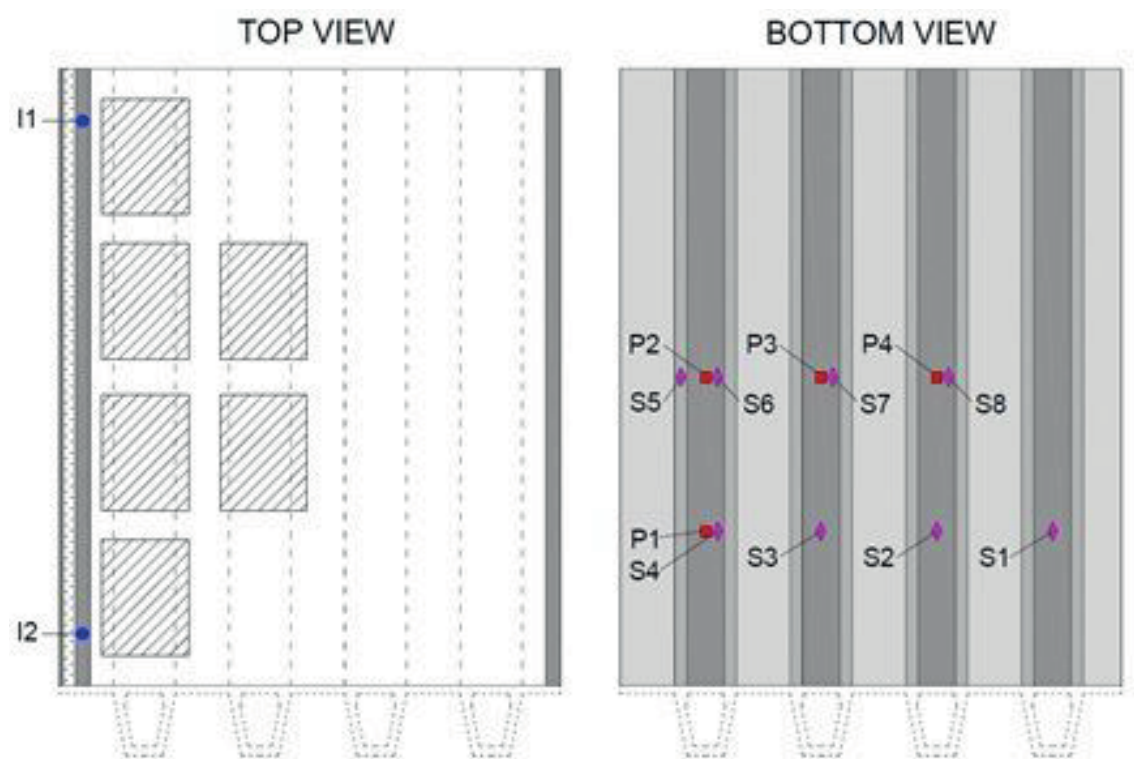

FIG. 9. Top and bottom views of the flyover. The truck configuration is shown along with the position of two inclinometers (I), four deflection prisms (P) and eight strain gauges (S). 


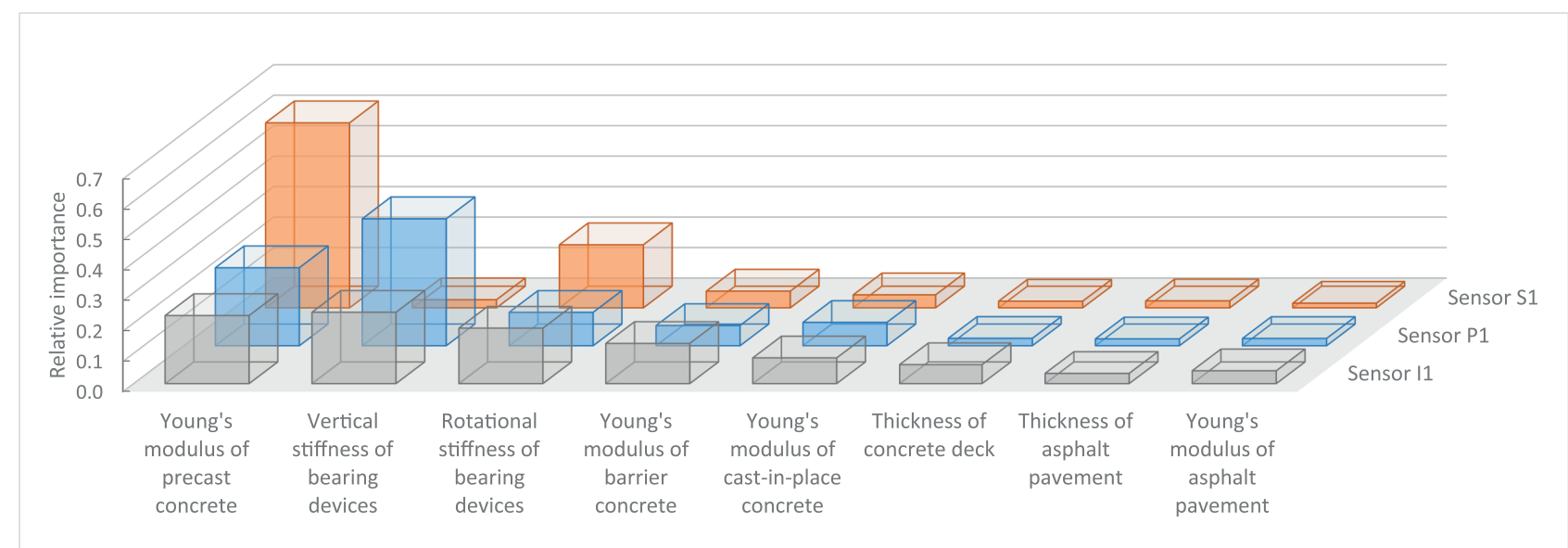

FIG. 10. Relative importance of parameters $\theta$ on model predictions at three sensor locations (P1, S1 and I1). 


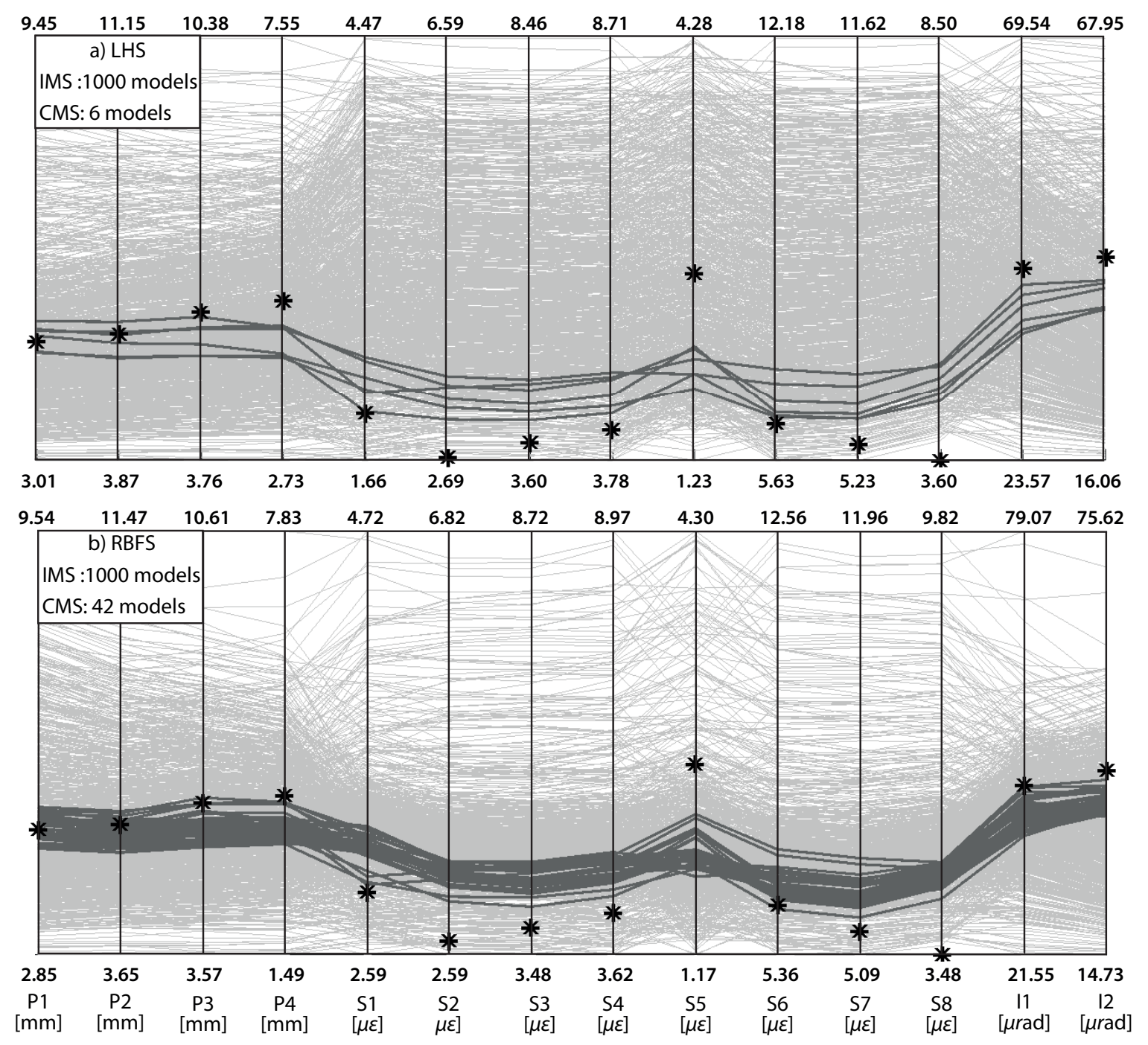

FIG. 11. Predictions of $\mathbf{1 0 0 0}$ model instances generated using Latin hypercube sampling (LHS), top and radial basis function sampling (RBFS), bottom. Each vertical axis represents the prediction at the sensor locations defined in Figure 9. Predictions of the initial model set (IMS) (grey lines) and the candidate model set (CMS) (dark lines) are plotted. CMS-prediction thresholds are reported for each axis. Black asterisks $\left(^{*}\right)$ represent the measured value at each location. 

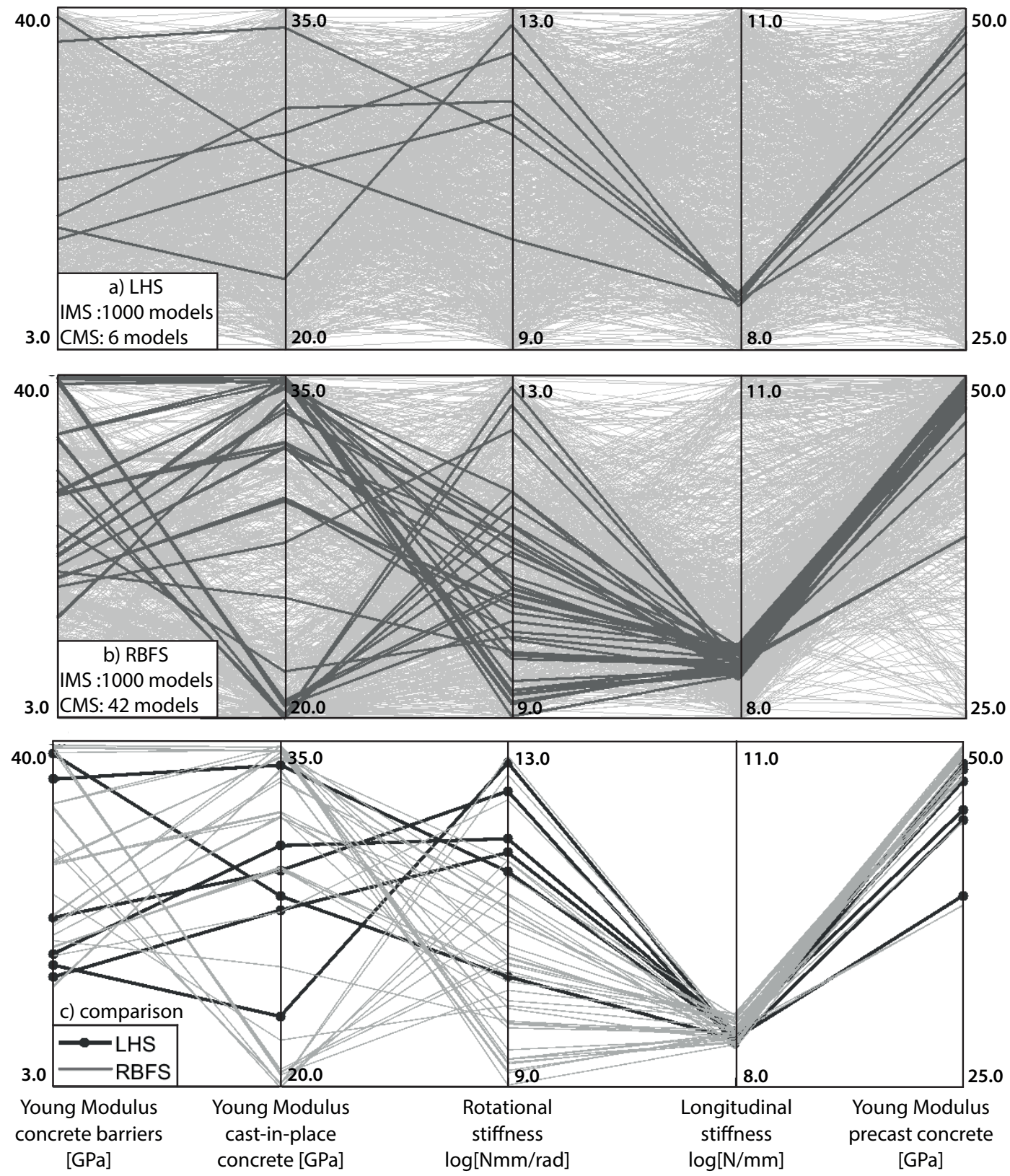

FIG. 12. Parameter values describing 1000 model instances generated using LHS and RBFS. Each vertical axis represents a parameter. Initial model instances (grey lines) and candidate models (dark lines) are plotted and initial values of each parameter are given for each axis. The comparison of candidate models is shown in c). 


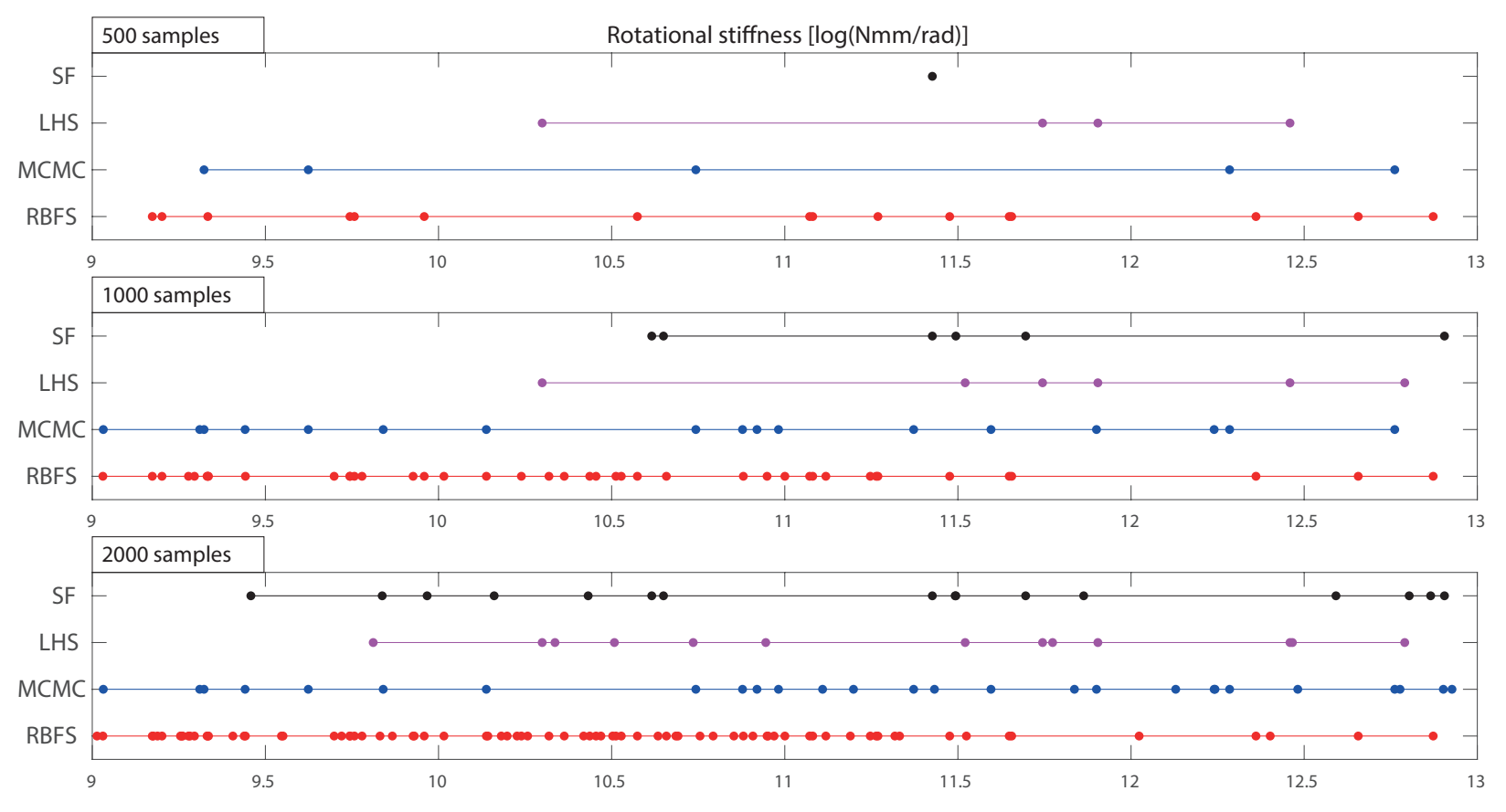

FIG. 13. Comparison of rotational stiffness values identified using RBFS, MCMC, LHS and SF. Falsification has been conducted considering three initial model sets that consist of 500, 1000 and 2000 samples. RBFS provides the largest CMS and the most effective exploration of the candidate domain, even with 500 samples. 


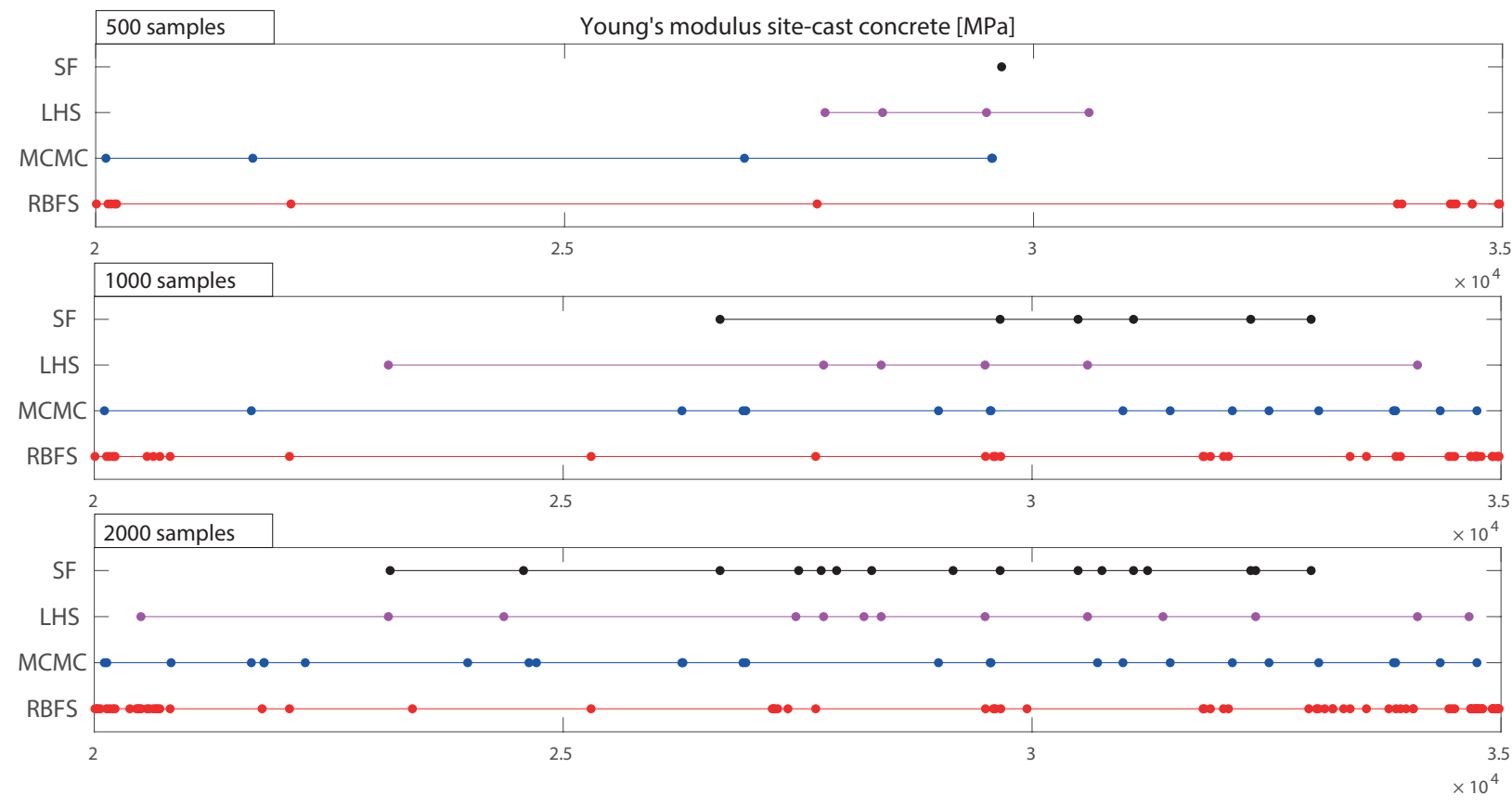

FIG. 14. Comparison of Young's modulus values for cast-in-place concrete, identified using RBFS, MCMC, LHS and SF. Falsification has been conducted considering three initial model sets that consist of 500, 1000 and 2000 samples. RBFS covers the entire candidate domain within $\mathbf{5 0 0}$ samples, performing better than MCMC. 


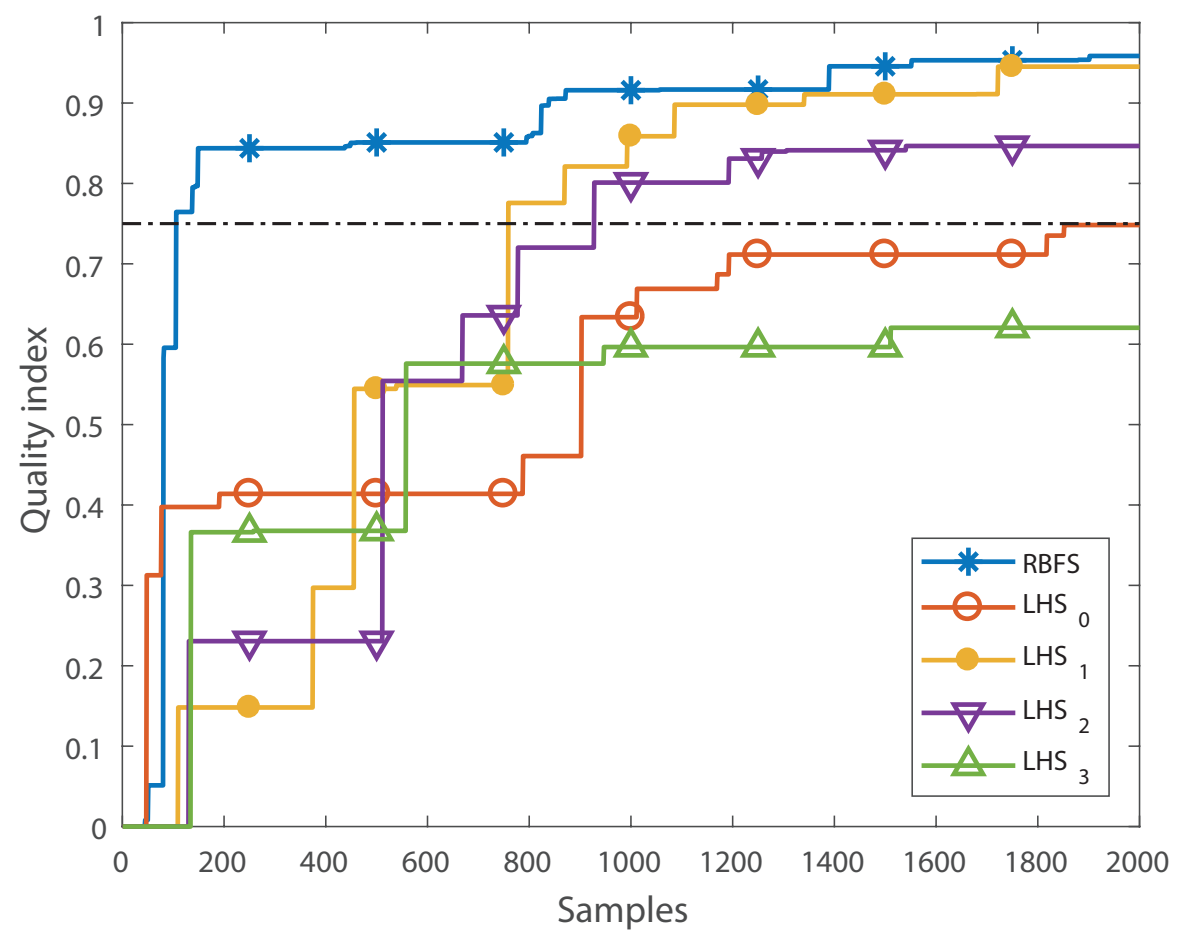

FIG. 15. Comparison of the candidate domain provided by 2000 samples generated using RBFS and four LHS. LHS $_{0}$ denotes the default LHS-setting implemented in ANSYS. Three more populations $\left(\mathrm{LHS}_{1}, \mathrm{LHS}_{2}\right.$ and $\mathrm{LHS}_{3}$ ) have been generated through a random selection of the sampling seed. The quality of candidate domain exploration provided by LHS is affected by the random choice of the seed value. 


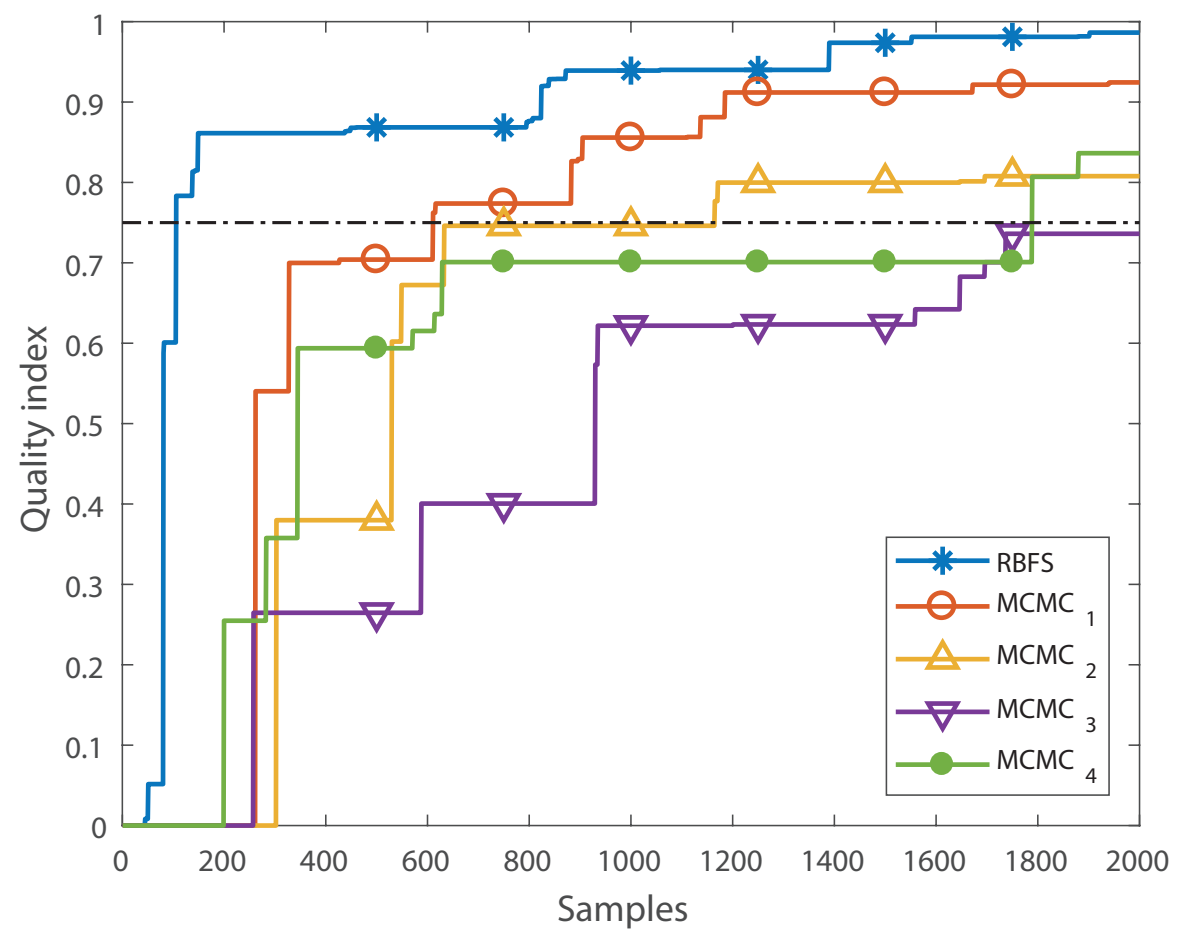

FIG. 16. Comparison of the candidate domain provided by 2000 samples generated using RBFS and four MCMC characterised by different proposal widths. MCMC $_{1}$ denotes the best parameter setting found after four attempts. Since RBFS does not require initial tuning, there is less risk of low-quality results. 


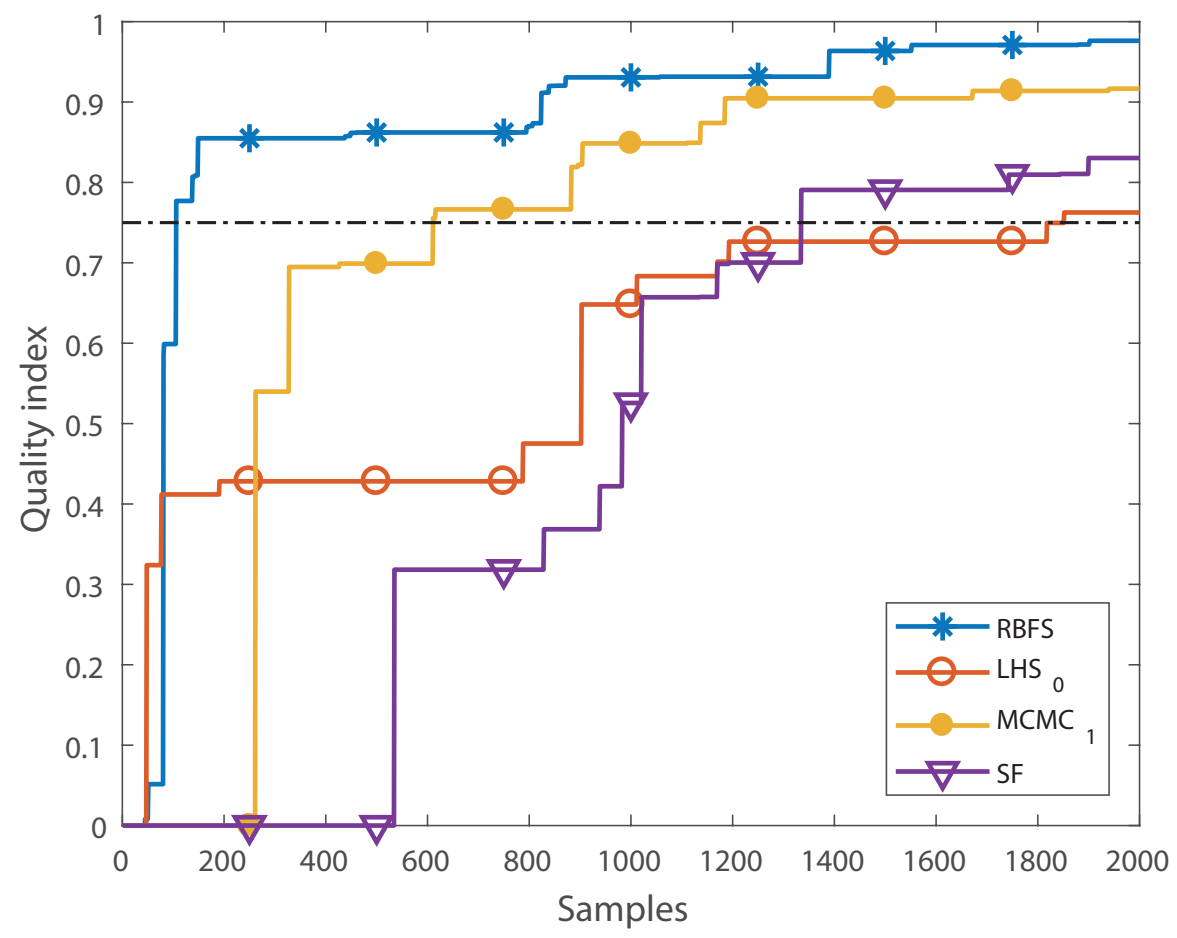

FIG. 17. Comparison of the candidate domain provided by 2000 samples generated using RBFS, $\mathrm{LHS}_{0}, \mathrm{MCMC}_{1}$ and SF. The main drawback of non-adaptive sampling is the slow exploration of the domain with increasing number of samples. 

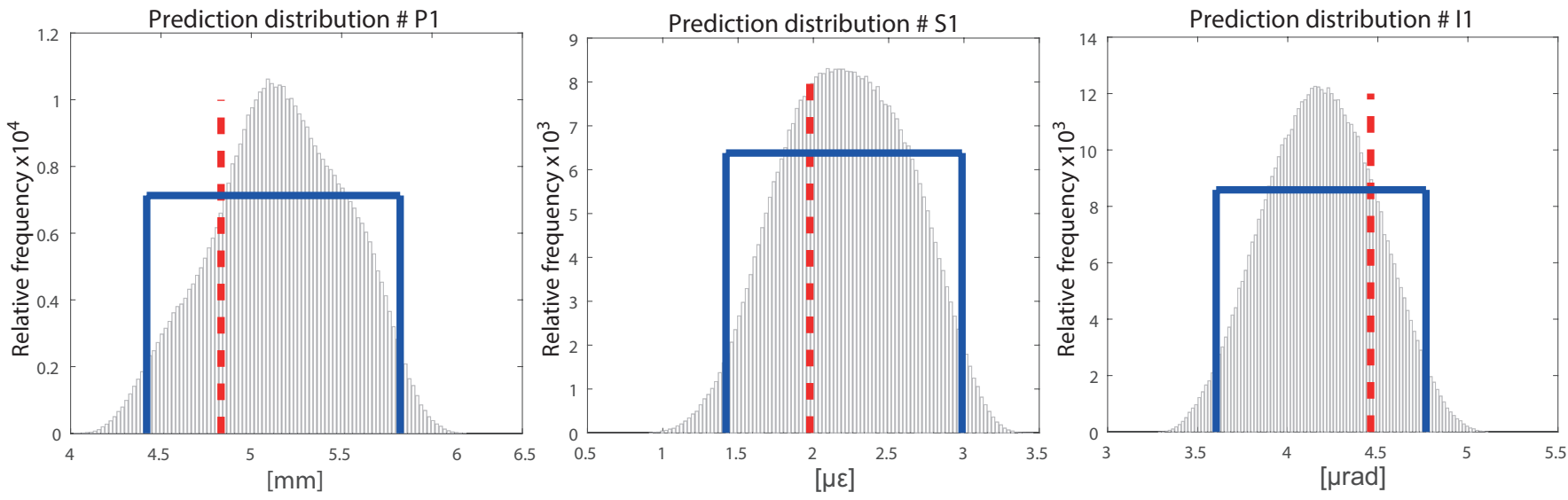

FIG. 18. Distribution of candidate-model-set predictions (PD) at three sensor locations (P1, S1 and I1) that have not been used for falsification. The uniform PDs for a $95 \%$ confidence and the CMS prediction distributions are plotted. For each sensor, the PD includes the measured value. The PDs have been identified using $\mathbf{2 0 0 0}$ initial samples generated through RBFS. 


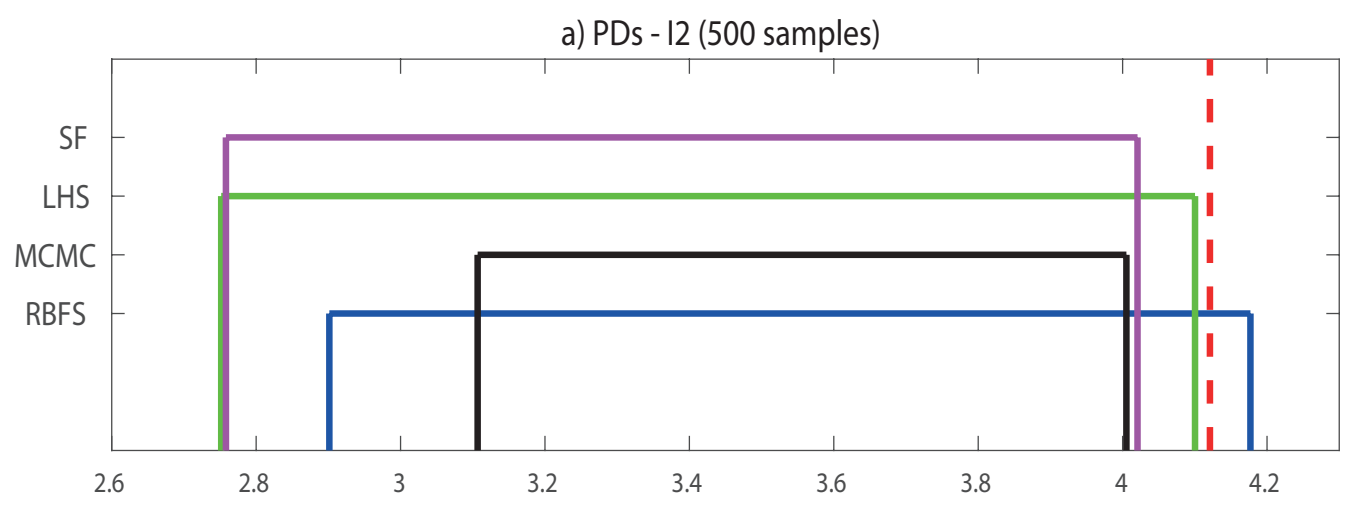

b) PDs - I2 (1000 samples)

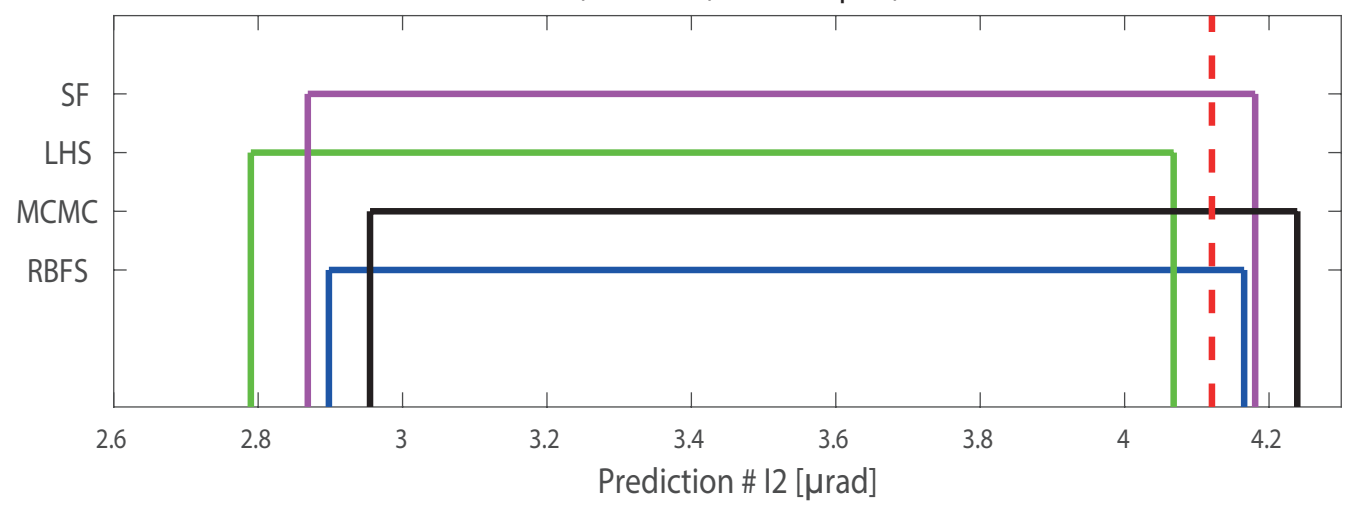

FIG. 19. Uniform distributions of candidate-model-set predictions (PD), for a $95 \%$ confidence, at sensor location 12 . Sensors P2, P3, P4, I1 and 12 have not been used for falsification and two PDs have been identified using respectively 500 (a) and 1000 (b) initial samples. The measurement value at location I2 is shown (dashed line). 